UCRL-8357

Physics and Mathematics

\author{
UNIVERSITY OF CALIFORNIA \\ Radiation Laboratory \\ Berkeley, California \\ Contract No. W-7405-eng-48
}

\title{
THE DEUTERON-PICKUP REACTION \\ IN AN OPTICAL-MODEL APPROXIMATION
}

Kenneth R. Greider

(Thesis)

July 7, 1958

Printed for the U. S. Atomic Energy Commission 


\section{DISCLAIMER}

This report was prepared as an account of work sponsored by an agency of the United States Government. Neither the United States Government nor any agency Thereof, nor any of their employees, makes any warranty, express or implied, or assumes any legal liability or responsibility for the accuracy, completeness, or usefulness of any information, apparatus, product, or process disclosed, or represents that its use would not infringe privately owned rights. Reference herein to any specific commercial product, process, or service by trade name, trademark, manufacturer, or otherwise does not necessarily constitute or imply its endorsement, recommendation, or favoring by the United States Government or any agency thereof. The views and opinions of authors expressed herein do not necessarily state or reflect those of the United States Government or any agency thereof. 


\section{DISCLAIMER}

Portions of this document may be illegible in electronic image products. Images are produced from the best available original document. 
This report was prepared as an account of Government sponsored work. Neither the United States, nor the Commission, nor any person acting on behalf of the Commission:

A. Makes any warranty or representation, express or implied, with respect to the accuracy, completeness, or usefulness of the information contained in this report, or that the use of any information, apparatus, method, or process disclosed in this report may not infringe privately owned rights; or

B. Assumes any liabilities with respect to the use of, or for damages resulting from the use of any information, apparatus, method, or process disclosed in this report.

As used in the above, "person acting on behalf of the Commission" includes any employee or contractor of the Commission to the extent that such employee or contractor prepares, handles or distributes, or provides access to, any information pursuant to his employment or contract with the Commission. 


\section{-is}

Contents

Abstract

Introduction

Part I. Formalism.

Section 1. Derivation of the transition-matrix element.

Section 2. Alternative derivation of the transition= matrix element.

Part II. Evaluation of the matrix element for central potential interactions.

Section 1. Derivation of optical-model wave functions.

Section 2. Evaluation of the cross section.

Part III. Comparison of the central potential theory with experiment.

Part IV. Evaluation of the matrix element with spin-orbit potentials included.

Section 1. Derivation of spin-orbit optical-model wave functions.

Sertion 2. Derivation of the spin matrix.

Section 3. Analysis of the polarization.

Part V. Comparison of the deuteron asymetry with experiment.

Appendix I. Inelastic scattering contributions.

Appendix II. Justification for Eq. (14), Part II. 
THE DEUTERON-PICKUP REACTION

IN AN OPTICAL-MODEL APPROXIMATION

Kenneth R. Greider

Radiation Laboratory

University of California

Berkeley, California

July 7, 1958

ABSTRACT

A theory of the $(p, d)$ pickup reaction is described, in which the nuclear interactions of the incoming and outgoing particles are considered. Two different formal expressions that give the transition amplitude are derived, and the wave functions in this amplitude are approximated by an optical-model procedure in which it is assumed that the initialo and final-state particles scatter elastically in the nucleus. The inelastic-scattering effects are shown to be small. Several closed forms for these optical-model wave functions are derived on the basis of a WKB approximation for a complex square-well scattering potential. The use of these wave functions, along with an approximation that gives the form of the transition amplitude in terms of Gaussian functions, allows a closed-form solution for the differential cross section.

It is found that the elastic scattering processes are not negligible, since they affect considerably the magnitude and the shape of the differential cross section. By comparing the theory with recent pickup experiments on $\mathrm{C}^{12}$ at 95 and $145 \mathrm{Mev}$, one obtains a nuclearmomentum distribution that, unlike the Born approximation analysis, is in good agreement with the results of other determinations of momentum 
distributions. It is found that a neutron number of from 4 to 7 neutrons and a momentum distribution of $\exp (-\mathrm{E} / 20)$ are required to fit the data. The polarization effects in the pickup process are investigated by using spin-orbit potentials in addition to the central potentials to describe the initialo and final-state scatterings. Simple forms for the splin ulblt, elastic scatteing wave functions are developed, so that the transition matrix can be obtained without difficulty in closed form. The usual polarimation formalism is applied to obtain the asymetry of deuterons produced by initially polarized protons, and the results compare favorably with recent experimental data. 


\section{INTRODUCTION}

The theory of nuclear-rearrangement collisions has been developed and refined to a considerable degree over the last ten years due to. both the "direct" interaction picture given by Serber $^{1}$ and the scattering formalism introduced by Lippmann and Schwinger. ${ }^{2}$ The two particular processes that have received perhaps the greatest amount of attention during this period are that of deuteron stripping, and its time reverse; deuteron pickup. Since the stripping process was observed first, and consequently received extensive experimental and theoreticail attention, a brief historical review of this subject is given in order to provide some insight into the problems that exist in the present treatment of the pickup process.

Excluding the very=low-cncrgy "Oppenhelmer'ePhililus effect," the first real nuclear stripping of medium- or high-energy deuterons was observed by Helmholz et al. in 1947.3 These reactions, in which various nuclei were bombarded with 190 $\mathrm{Mev}$ deuterons, produced highenergy neutrons whose angular distributions were peaked in the forward direction. This peak could not be explained on the basis of a compoundnucleus theory, which would lead to a flat angular distribution, but required Serber's direct-interaction picture. 4

The Serber theory considers basically the kinematic effects when the deuteron's kinetic energy is much greater than its binding energy. The proton ${ }^{*}$ in the deuteron may be instantaneously stripped

This particular case is chosen only for clarity in the discussion that follows. The word "neutron" may be substituted for "proton" and vice versa, since either neutrons or protons may emerge from stripping reactions. 
off by the target nucleus, while the neutron continues on with the momentum it had when the proton was removed. This momentum is just the vector sum of:

(a) onemalf the nriginal deuteron translational momentum, and

(b) the internal momentum of the neutron in the deuteron at the instant of stripping.

Since this internal momentum is randomly oriented, and is much smaller than the translational momentum, the predicted distribution of neutrons is highly peaked in the forward direction and gives a successful explanation of the form of the experimental results.

To obtain an estimate of the magnitude of the stripping cross section, the Serber theory uses a classical model. Consider a hard spherical nucleus bombarded by a deuteron in which classical trajectories are assumed for both the neutron and proton. The nuclear interaction is simply represented, since those trajectories that cross the nuclear surface represent capture processes. Thus stripping can occur only if the neutron misses the nucleus and the proton is captured. At the high energy considered, such a classical picture gives reasonably good results for the total cross section.

In spite of the successes of the serber theory at high energies, it could not explain the angular distributions obtained a few years later by Burrows et al. ${ }^{5}$ By the stripping of medium-energy deuterons, they obtained some neutrons whose angular distributions peäked at wide angles rather than in the forward direction. This phenomenon was explained by Butler ${ }^{6}$ on the basis of a model apparently quite different from Serber's。 
Very briefly, Butler's theory assumes that the proton is captured into a nuclear state of definite angular momentum and parity. Consider, for example, the stripping process that leaves the nucleus in a final ground state (which is experimentally known by resolving individual energy groups of the observed neutrons). The initial nucleus, then, can accept only those protons which carry the particular angular momentum required to form a ground-state final nucleus; and consequently the angular distribution of the outgoing neutrons will be drastically affected. The fact that one can experimentally observe the angular-momentum difference between initial and final ground-state nuclei has provided a powerful tool in the field of nuclear spectroscopy.

The Butler theory does not, of course, negate any of the Serber theory; it merely takes up the prohlem in greater dotall. Firot; Butler treats the problem by quantum mechanics rather than by a classical approximation, and secondly, he places restrictions on the possible final states of the captured proton, rather than assume that all protons are captured, regardless of their momenta. One might assume less severe restrictions than Butler's, such as requiring that the captured protons have momentum values that coincide with the known nucleon momentum distribution in an nucleus. But this falls into the same category as Butler's angular-momentum requirements, in that it takes account of the restrictions on the momentum of the captured particle due to the presence of a real nucleus, rather than a classical absorbing sphere.

Although present experimental evidence does not show the necessity for any drastic revisions in stripping theory, there are improvements, to be discussed below, that can be made on the Serber and Butler 
results. There have recently been several investigations, $7,8,9,10$ based on scattering formalism, which obtain more exact results than the earlier theories. Comprehensive reviews of the current status of stripping reactions have been written by Huby ${ }^{11}$ and by Butler. 12

In extending the Butler theory, one might wonder whether the nucleus has any appreciable effect on the incoming deuteron or the out going neutron, in addition to its effect on the state of the captured proton discussed above. Certainly one mould expect these particles to scutter frum the nuclcus and ohange the observed angular distributions. The Butler theory assumes that the nucleus is completely transparent both to incoming deuterons and to outgoing neutrons. This presumably accounts for the fact that the Butler cross sections are larger than the experio mental results, since the nucleon mean free path at Butler's energies is small compared with nuclear dimensions, and absorption effects should not be negligible. At high energies, where the nucleon mean free pall is long, it would be expected that the nucleon-nucleus scattering contributions would not only affect the magnitude of the cross section, but also alter the angular distribution of the observed stripped neutrons. To include the aforementioned scattering contributions, and give an exact treatment of the stripping reaction, is by no means easy. The first step, to derive an exact formal expression that is believed to represent the process, is made possible with the results of the Lippmann-Schwinger formalism. However, the exsct calculation of such an expression is usually impossible. The reason for the difficulties can be explained as follows. In the Born-approsimation treatment (Butler and Serber) not only is the mathematical description of the plane-wave particle states well known, but also their form usually 
permits quite simple and direct evaluation of the desired cross sections. With the inclusion of initial and final scattering states, however, the simple Born-approximation description is no longer applicable, and one must either use higher orders in Born approximation, or, better, describe the distorted states by means of the results of multiple scattering theory. ${ }^{13}$ In either case, there is the additional mathematical problem of evaluating the matrix elements, since the integrals that appear are usually quite formidable. Thus it would be desirable to find a formal description of the scattering of particles that is reasonably exact, and at the same time not too difficult to handle mathematically.

Among the various methods that aim for this goal, the opticalmodel approach of Fernbach, Serber, and Taylor ${ }^{14}$ appears to be a good approximation to use in rearrangement collisions. The elastic scattering is described in terms of a complex potential, in which the real part give's the attraction or repulsion of the nuclear well, and the imaginary part accounts for the loss of particles due to all inelastic processes. (If this method is used, one must show that the inelastic processes comprise a small part of the total scattering contributions to the matrix element.) This approach to the stripping problem has been suggested by Francis and Watson, 8 and calculations for low-energy processes have been made by Cheston, ${ }^{15}$ who also considered polarization eppects.

All the arguments of the preceding paragraphs are equally applicable to the deuteron pickup process. For the sake of clarity, the specific process under consideration is that in which a proton picks up a bound neutron to form a deuteron, leaving the final nucleus in the ground state or in a low excited state. 
Chew and Goldberger ${ }^{16}$ have investigated the pickup process in Born approximation and obtained a result somewhat similar to the Butler theory. Their differential cross section depends on two factors:

(a) the probability that the neutron to be picked up has a momentum $\vec{k}_{\mathrm{N}}$ in the initial nucleus, and

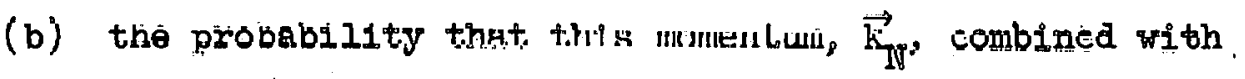
the incident proton momentum through the mutual interaction, $\mathrm{V}_{\mathbb{N P}}$ '. can be found in a deuteron.

The nnly unknown parameter in this theory is the neutron momentum $\vec{k}_{N}$ 。 Thus by fitting the theory to experimental angular distributions, one can find the probability distribution of neutron momenta in the initial nucleus.

By describing the nuclear scattering of the incident proton and outgoing deuteron, the optical model referred to above adds a third factor to the Chew Goldberger expression for the differential cross Dection: the probability that the incident proton (outgoing deuteron) has elastically scattered into a different momentum state before (after) the neutron is picked up. Implicit in this factor is the probability that the particle may be absorbed by the nuclear medium.

In view of the discussion above, it is apparent that the exact solution of the deuteron-pickup problem is virtually impossible to obtain, and that the optical-model approximation might make the problem tractable. Such an optical-model description has been used in this dissertation.

The major goals of the work to be described here were:

(a) to derive an exact formal expression that describes the pickup reaction; 
(b) to that the nuclear scattering contributions from the incoming and outgoing particles cannot be neglected in an accurate calculation of the cross section and polarization effects in the process;

(c) to verify the optical-model approach as an adequate approximation for these scattering contributions; and

(d) to develop manageable optical-model wave functions, and suggest some techniqued for evaluation of the lintegrals. It is assumed in the following discussion that the energy of the incident proton is high, so that surface reflection and refraction, as well as the effects of the nuclear Coulomb potential, can be neglected.

Part I shows the development of two forms for the matrix element that describes the pickup process and includes a discussion of the merits of each. The optical-model restriction that the incoming and outgoing particles scatter coherently is imposed, and it will be shown that the Chew-Goldberger expression can be obtained as a special case of the more general matrix element presented.

General forms for the wave functions that can be used to describe the elastic scattering in a central nuclear potential are developed in Part II. The derivation is based on a WKB approximation to obtain results that are as easy to handle mathematically as the Born approximation's plane waves. Finally the differential cross section is ob. tained in closed. form by using Gaussian expressions to approximate the functions in the matrix element.

In Part III, calculations are made to fit the theory with recent pickup experiments on $\mathrm{c}^{12} \cdot 17,18$ It is shown that by the inclusion of : particle interactions with the nucleus, a fit can be obtained without using the high neutron momenta that are required in the Chew $₫$ Goldberger 
analysis. The momentum distribution actually obtained for $\mathrm{C}^{12}$ is of the form $\exp (-E / 20)$, which is in agreement with the results of other investigations.

Furthermore, since the optical model accounts for particle absorption, Part III shows that a reasonable value is obtained for the magnitude of the cross section. The integral of the momentum distribution over all neutron momenta gives the number of neutrons in the nucleus that contribute to the pickup process. The number obtained in the present analyoic is on the orier nf a 1,1 the neutrons in the nucleus, or perhaps, all the neutrons in the $\mathrm{p}$ shell. This is to be compared with the value of 0.06 obtained from the Born approximation. ${ }^{17}$ The curves that describe these results are found in Fig. 1.

The posstbility of polarization effects in pickup reactions is a unique prediction of a theory, ${ }^{19}$ such as the optical model, that treats the nuclear scattering and absorption of initial- and flial-state particles. Cheston has used the optical model to calculate the polarization in low-energy stripping reactions, ${ }^{15}$ and obtains results that are in reasonable agreement with experiment. 20 other less successful models that obtain polarization effects in stripping and pickup reactions have been suggested by Newns ${ }^{21}$ and others. 22,23,24.'Part IV is devoted to a derivation of elastic-scattering wave functions resulting from the addition of a spin-orbit potential to the central potential. Like the central-potential wave functions developed in Part II, these spin-orbit wave functions have forms that permit easy evaluation of the integrals. The analysis of the polarization is carried out by using the $M$ matrix formalism of Wolfenstein and Ashkin. 25 
The results of the polarization analysis are applied to a specific case in Part V. The asymmetry of the outgoing deuterons produced by incident polarized protons is calculated, and the results are compared with recent experimental data ${ }^{18}$ in Fig. 2. 


\section{FORMALISM}

Section 1. Derivation of the Transition-Matrix Element

In order to obtain information on the cross section, energy, spin, etc. of the deuterons produced from nuclei bombarded with protons, it is most convenient to use the scattering formalism introduced by Lippmann and Schwinger. ${ }^{2}$ This formalism provides a method for obtaining the transitionmatrix elements which contain the desired intormation abuut the final-state deuterons in terms of the initial conditions (momentum, spin, etc.) on the incoming protonmucleus system. It is necessary first to explicitly define the noninteracting initial and final states in order to describe the operations that later lead to the transition matrix.

Consider an initial state consisting of a proton, whose momentum is $\overrightarrow{h k}_{0}$ in the center ofomass system, incident on a nucleus which is described by a wave function $x_{0}\left(\vec{\tau}_{A}\right)$. Here $\vec{\tau}_{A}$ represents the coordinates of the A nuclear particles, and the subscript " $O$ " designates a nucleus in its ground state. For clarity of notation, all spin indices have been suppressed for the present. The wave function for the proton-nucleus system,

$$
\varphi_{i}\left(\vec{\tau}_{A}, \vec{r}_{P}\right)=x_{O}\left(\vec{\tau}_{A}\right) e^{i \vec{k}_{0} \cdot \vec{r}_{P}},
$$

is the solution of the Schroedinger wave equation,

$$
\left(\mathrm{H}_{\mathrm{O}}+\mathrm{T}_{\mathrm{N}}+\mathrm{T}_{\mathrm{P}}+\mathrm{V}_{\mathrm{N}}\right) \varphi_{i}\left(\vec{\tau}_{\mathrm{A}}, \vec{r}_{\mathrm{P}}\right)=\mathrm{E}_{\mathrm{i}} \varphi_{\mathrm{i}}\left(\vec{\tau}_{\mathrm{A}}, \overrightarrow{\mathrm{r}}_{\mathrm{P}}\right)
$$

$\mathrm{H}_{\mathrm{O}}$ is the total Hamiltonian for $\mathrm{A}-1$ nucleons, and $\mathrm{T}_{\mathrm{N}}$ and $\mathrm{T}_{\mathrm{P}}$ are the kinetic energy operators for the neutron and proton respectively. The neutron to be picked up is bound to the core of A-I nucleons by the potential $\mathrm{V}_{\mathrm{N}^{\circ}}$ 
The final state, which consists of a free deuteron of momentum 后, and $A-1$ nucleons in some nuclear state $n$, is described by the wave function

$$
\varphi_{f}\left(\vec{\tau}_{A}, \vec{r}_{P}\right)=\chi_{n}\left(\vec{\tau}_{A-1}\right) \phi_{D}(\vec{r}) e^{i \vec{K} \cdot \vec{R}},
$$

which is the solution of

$$
\left(H_{O}+T_{N}+T_{P}+V_{N P}\right) \varphi_{f}\left(\vec{r}_{A}, \vec{r}_{P}\right)=E_{f} \varphi_{f}\left(\vec{\tau}_{A}, \vec{r}_{P}\right) ;
$$

$\varnothing_{D}(\vec{r})$ is the wave function of the bound deuteron, and $V_{N P}$ is the neutronproton potential. The deuteron center-of-mass coordinate $\vec{R}$ and the relative coordinate $\vec{r}$ are related to the neutron and proton coordinates by the relation.

$$
\begin{aligned}
& \vec{R}=\frac{\vec{r}_{P}+\vec{r}_{N}}{2}, \\
& \vec{r}=\vec{r}_{P}-\vec{r}_{N} .
\end{aligned}
$$

The total wave function $\Psi$ which describes the complete interacting proton-nucleus system, is the solution of the wave equation involving the total Hamiltonian, $\mathrm{H}$ :

$$
H \Psi=\left(H_{O}+T_{N}+T_{P}+V_{N}+V_{P}+V_{N P}\right) \Psi=E_{i} \Psi
$$

The potential $\mathrm{V}_{\mathrm{P}}$ of Eq. (6) describes the interaction of the proton with the $A-1$ core nucleons. If the total wave function $\Psi$ is expressed in terms of the incoming free state of $\mathrm{Eq}_{\circ}$ (I) plus a scattered wave, the transition matrix may then be obtained by picking out final states of outgoing deuterons from the scattered wave.

Followiing Lippmann and Schwinger, we-can write the expression for the solution of $\mathrm{Eq} \cdot(6)$ corresponding to incoming plane waves and 
outgoing spherical waves:

$$
\psi^{(+)}=8^{(+)} \varphi_{1}=\left[1+\frac{1}{a^{(+)}+V_{N}-V_{P}-V_{N P}}\left(V_{P}+V_{N P}\right)\right] \varphi_{i},
$$

where the notation has been condensed by setting

$$
a^{(+)}=E_{i}-H_{n}-T_{N}=T_{H}+i f .
$$

How $\mathrm{Eq} .(7)$ is not in a suitable form for the application of the elastic scattering approximations we wish to make. We may rewrite Eq. (7) as

$$
\psi^{(t)}=\varphi_{i}+\frac{1}{a^{(t)}-V_{N P}-V_{N}} V_{N P} \varphi_{1}+\frac{1}{a^{(t)}-V_{N P}-V_{N}} V_{P} \psi^{(t)}
$$

and verify that it satisfies Eqs. (2) and (6) by direct substitution. The Green's function in Eq. (9) represents outgoing deuterons in which the neutron interacts with the core through the potential $\mathrm{V}_{\mathrm{N}^{\circ}}$ We can therefore define a wave function $\Phi_{N}$ to describe this,

$$
\Phi_{N}^{(\omega)}=\delta_{\mathbb{N}}^{(\omega)} \varphi_{f}=\varphi_{f}+\frac{1}{a^{(\sigma)}-V_{N P}} V_{N} \Phi_{N}^{(m)}
$$

where

$$
\Omega_{N}^{(\infty)}=1+\frac{1}{a^{(\infty)}-V_{N P}-V_{N}} V_{N}
$$

Now by expanding $\left(a^{(+)}-V_{\mathbb{N P}}-V_{\mathbb{N}}\right)^{-1}$ of $\mathrm{Eq}$. (9) in the usual way in outgoing deuteron states (in which the neutron interacts with the core), we obtain the exact transition-matrix element for the pickup process:

$$
\mathrm{T}_{f i}=\left(\Phi_{\mathrm{N}}^{(\omega)}\left|\mathrm{V}_{\mathrm{NP}}\right| \varphi_{i}\right)+\left(\Phi_{\mathrm{N}}^{(\varphi)}\left|\mathrm{V}_{\mathrm{P}}\right| \psi^{(+)}\right)
$$

It should be noted that no approximations have been used in deriving Eq. (12). This transition amplitude, however, is not a very tractable 
expression as yet, since only the functional forms of $V_{N P}$ and $\varphi_{1}$ are reasonably well known. Thus we must make some approximations for $\Phi_{N}^{(\omega)}$, $\mathrm{V}_{\mathrm{P}}$, and $\psi^{(+)}$in order to evaluate $\mathrm{Eq}$. (12).

The first approximation concerns $V_{P}$ and $\psi^{(t)}$. We note that by setting $V_{N}=V_{P}=0$ in $E q$. (12) we are left with only the $V_{N P}$ term in Born approximation. This term, however, gives exactly the Chew-Goldberger expression (or the Butler expression for the stripping process). owing to the successes of these theories in explaining the gross features of the processes, and because of the physical argument that $V_{N P}$ should represent the main interaction for pickup (since the neutron and proton must "see" each öther in order to form a deuteron), we are led to look for a solution to Eq. (12) that is exact in terms of $V_{N P}$ and approximate with respect to $V_{P}$ and $V_{N}$. We replace the last term of Eq. (12) by an expression that describes only elastic or coherent scatterings of the proton in the nucleus:

$$
\left.\left.\mathrm{v}_{\mathrm{P}} \mid \psi^{(+)}\right) \simeq v_{\mathrm{CP}} \mid \Omega_{\mathrm{CP}}^{(+)} \varphi_{i}\right)
$$

where

$$
\delta_{\mathrm{CP}}^{(+)}=1+\frac{1}{\mathrm{a}^{(+)}-v_{\mathrm{N}}-v_{\mathrm{CP}}} v_{\mathrm{CP}},
$$

and $V_{\mathrm{CP}}$ and $\Omega_{\mathrm{CP}}^{(+)}$are defined to be diagonal with respect to the energy of the core. $V_{\mathrm{CP}}$ is a potential describing only elastic proton-nucleus interactions, and can be deduced from multiple scattering theory, or may be replaced by the phenomenological optical potential of Fernbach, Serber, and Taylor. 14 The importance of the neglected inelastic wave contributions in Eq. (13) is discussed in Appendix I. 
Since, in Eq. (14), $a^{(+)} \cdot \mathrm{V}_{\mathrm{N}}$ is diagonal and represents states of all a nucleons, $V_{\mathrm{CP}}$ then gives the elastic proton interactions with all A nucleons. It might be argued therefore that to make Eq. (13) more exact, we should have an additional term $\cdot V_{N P} \psi^{(t)}$ on the left side, since $V_{P}$ includes the proton interaction with only $A-1$ nucleons. We can Just1fy Eq. (13) by expending $\psi^{(+)}$and ceeing that the proton interacts through $\mathrm{V}_{\mathrm{P}}+\mathrm{V}_{\mathrm{NP}}$ for all but the last scattering, in which $\mathrm{V}_{\mathrm{NP}}$ is absent (1.e., the neulrun does not intcract). If $A$ is large we can assume that the omission of the neutron from the $A$ nucleons in the last scattering will not affect the elastic description of the proton scattering. Substituting Eq. (13) into (12), we obtain the transition matrix

$$
T_{f^{i}}=\left(\Phi_{\mathbb{N}}^{(-)}\left|v_{N P}\right| \varphi_{i}\right)+\left(\Phi_{\mathbb{N}}^{(-)}\left|v_{C P}\right| \delta_{C P}^{(+)} \varphi_{i}\right)
$$

We are now in a position to condense Eq. (15) into a more compact expression. We note from Eqs. (2), (4), (8), (10), and (14) the relations

$$
\begin{aligned}
& \left(a^{(-)}-v_{N P}-v_{N}\right) \delta_{N}^{(-)} \varphi_{f}=-i \epsilon \varphi_{f}, \\
& \left(a^{(+)}-v_{N}\right) \varphi_{i}=i \epsilon \varphi_{i}, \\
& \left(a^{(+)}-v_{N}-v_{C P}\right) \delta_{C P}^{(+)} \varphi_{i}=1 \in \varphi_{i}
\end{aligned}
$$

In Eqs. (16), we have used $E_{f}=E_{i} \cdot$. Using (16a) and (16b) in the first term of Eq. (15), we obtain

$$
\left(\Phi_{N}^{(-)}\left|V_{N P}\right| \varphi_{i}\right) \doteq 1 \in\left(\Phi_{N}^{(-)} \mid \varphi_{i}\right)=i \in\left(\varphi_{f} \mid \varphi_{i}\right) .
$$


Likewise, using (16c) and (16a) in the second term of Eq. (15), we obtain

$$
\begin{gathered}
\left(\Phi_{\mathbb{N}}^{(\infty)}\left|v_{\mathrm{CP}}\right| \delta_{\mathrm{CP}}^{(+)} \varphi_{i}\right)=\left(\Phi_{\mathrm{N}}^{(-)}\left|v_{\mathrm{NP}}\right| \delta_{\mathrm{CP}}^{(+)} \varphi_{1}\right)+i \epsilon\left(\varphi_{f} \mid \delta_{\mathrm{CP}}^{(+)} \varphi_{i}\right) \\
-i \in\left(\Phi_{\mathbb{N}}^{(-)} \mid \varphi_{i}\right) \quad \cdots
\end{gathered}
$$

Substituting Eqs. (17) and (18) into (15), we find

$$
T_{f i}=\left(\delta_{N}^{(-)} \varphi_{f}\left|v_{N P}\right| \delta_{C P}^{(t)} \varphi_{i}\right)+i \epsilon\left[\left(\varphi_{f} \mid \delta_{C P}^{(t)} \varphi_{i}\right)-\left(\varphi_{f} \mid \varphi_{i}\right)\right],
$$

or

$$
\mathrm{T}_{f i}=\left(\delta_{\mathrm{N}}^{(-)} \varphi_{f}\left|\cdot \mathrm{V}_{\mathrm{NP}}\right| \delta_{\mathrm{CP}}^{(+)} \varphi_{\dot{I}}\right)
$$

In Eq. (19) we have omitted the term involving the factor if since, as we will now show, its contribution is zero:

$$
i \in\left[\left(\varphi_{f} \mid \Omega_{\mathrm{CP}}^{(+)} \varphi_{i}\right)-\left(\varphi_{f} \mid \varphi_{i}\right)\right]=i \in\left(\varphi_{f}\left|\frac{1}{a^{(+)}-v_{N}-v_{C P}} v_{C P}\right| \varphi_{i}\right) .
$$

We expand $\left(a^{(+)}-v_{N}-v_{C P}\right)^{-1}$ in a complete set of states:

$$
\left(a^{(+)}-v_{N}-v_{C P}\right)^{m I}=\sum_{k} \frac{\psi_{k}><\psi_{k}}{E_{i}-E_{k}+1 \epsilon},
$$

where $\psi_{k}$ can be written in the form $\psi_{k}=\dot{x}_{0}\left(\vec{t}_{A}\right) \varphi_{P}(k)$ because $v_{C P}$ requires that $\psi_{\mathrm{k}}$ be diagonal in the energy of the nuclear part of the wave function. When the sumation is replaced by an integral, Eq. (21) becomes

$$
\left(a^{(t)}-v_{N}-v_{C P}\right)^{-1}=\int d E_{k} \frac{\left|x_{0}\left(\vec{\tau}_{A}\right) \varphi_{P}(\vec{k})>\alpha_{0}\left(\vec{\tau}_{A}\right) \varphi_{P}(\vec{k})\right|}{E_{i}-E_{k}+i \epsilon} .
$$


Substituting $\mathrm{E} \dot{q}_{0} \cdot(22)$ into $\mathrm{Eq} \cdot(20)$, we obtain

$$
\begin{aligned}
& 1 \in\left[\left(\varphi_{f} \mid \delta_{\mathrm{CP}}^{(+)} \varphi_{i}\right) \propto\left(\varphi_{f} \mid \varphi_{i}\right)\right]= \\
& =i \epsilon \int d E_{l k}\left(\varphi_{f} \mid x_{0}\left(\vec{\tau}_{A}\right) \varphi_{P}(\vec{k}) \frac{l}{F_{i}-E_{k}+i \epsilon}\left(x_{O}\left(\vec{g}_{A}\right) \cdot \varphi_{P}(\vec{k})\left|v_{C P}\right| \varphi_{i}\right) .\right.
\end{aligned}
$$

For $E_{k} \neq E_{i}$, the expression on the right vanishes in the limit of $\epsilon \rightarrow 0$ because of the factor of $\epsilon$ in the numerator. For $E_{k}=E_{i}$, there is no contribution to the integral from the one polnt $E=\mathbb{E}_{1}$ unlece the numerator contains separate delta functions in the neutron and proton energy. (See discussion following Eq. (31)!) Since the neutrons change from a bound nuclear state to a free state in a deuteron, such a delta function is not possible, and the term is asymptotically zero. Thus Eq. (19) holds, and verifies a somewhat different derivation by Francis and Watson. 8

our second elastic-scattering approximation is to rejlace $\Phi_{N}^{(-)}$, defined by $\mathrm{Eq} .(10)$, by $\Phi_{\mathrm{CN}}^{(-)}$, where

$$
\Phi_{\mathrm{CN}}^{(\infty)}=\delta_{\mathrm{CN}}^{(\infty)} \varphi_{\mathrm{f}}
$$

describes a wave of deuterons in which the neutron interacts elastically with the core nucleons, and where

$$
\delta^{(\infty)} \mathrm{CN}=1+\frac{1}{a^{(-)}-V_{\mathrm{NP}}-U_{\mathrm{CN}}^{\dagger}} v t_{\mathrm{CN}} .
$$

Here $\delta^{(-)} \mathrm{CN}$ and $v_{\mathrm{CN}}$ are optical-model operators similar to $\Omega^{(+)} \mathrm{CP}$ and $v_{\mathrm{CP}}$, discussed after Eq. (14). We finally obtain the transition matrix, 


$$
\mathrm{T}_{f i}=\left(\delta^{(-)} \mathrm{CN} \varphi_{f}\left|\mathrm{v}_{\mathrm{NP}}\right| \delta_{\mathrm{CP}}^{(+)} \varphi_{i}\right) \ldots
$$

As with the proton wave function, it is assumed here that the incoherent waves in the final-state wave function $\Phi_{N}^{(\infty)}$ can be neglected. There are several qualitative arguments to support this: First, the Pauli exclusion principle forbids "hard" nucleon nucleon scatterings in a nucleus except for those thist leave the particle in unoccupied momentum states beyond the Fermi sphere. Consequently, the inelaștic collisions that do occur must be associated with large momentum transfers at large scattering angles, and can thus be experimentally separated from elastic effects which describe the scattering at small angles. Secondly, the experimental fact that the pickup process usually leaves the final nucleus in its ground state gives a strong argument for neglecting inelastic scatterings. For a particle incident on a nucleus in its ground state, there are many channels open for inelastic scatterings to excited states where the density of states is large, but there are few channels back to the ground state. Thus it is improbable that an inelastically scattered particle will find its way back to a state of low or zero excitation. Finally, one may argue that the inelastic scattering of the outgoing deuteron will not contribute to the pickup cross section, since it tends to break up the deuteron. This is because such inelastic effects arise from "hard" scatterings of the individual neutron and proton in the deuteron. Unless these two particles scatter coherently in "hard" interactions, the small deuteron binding energy should not be expected to keep them together. A more rigorous discussion of these arguments is found in Appendix $I_{2}$ and it will be assumed in the rest of this thesis that, for the purposes of describing the pickup reaction, the elasticescattering approximations give an adequate and reasonably accurate description of the true scattering. 


\section{Section 2. Alternative Derivation of the Transition-Matrix Element}

Although the transition matrix of Eq. (25) can now be used to obtain the desired information about the outgoing deuteron states, there are, however, two objections that may be raised concerning its form:

(a) Within the Iimits of thr uphlical-model approximatione of Section 1, Eq. (25) is still not an exact expression, owing to the additional approximation that negglects the lasl $V_{N P}$ interaction in the scattering of the incoming proton from the A nucleons (see discussion preceding Eq. (15));

(b) the final-state wave function defined in Eqs. (10) and (23) appears rather unsymmetric, in that it represents an outgoing deuteron in which only the neutron interacts with the residual nucleus. It would be desirable, then, to find a new form for the transition matrix which requires no approximations beyond the optical-model assumptions, and rcprcocnte the outgoing denternn state so that both the neutron and proton interact symmetrically with the residual nucleus. 26 An expression that fulfills both of these requirements has been partially developed by Gell-Mann and Goldberger ${ }^{9}$ but it will be shown to be derivable from Eq. (12) above.

We define a wave function $x^{(+)}$representing an incoming proton wave in which the proton interacts with the core only through the potential $\mathrm{V}_{\mathrm{P}}$ :

$$
x^{(t)}=\varphi_{i}+\frac{I}{a^{(t)}-v_{N}} v_{P} x^{(t)} .
$$

Here $x^{(t)}$ is related to the total wave function $\psi^{(t)}$ by the integral equation 


$$
\psi^{(t)}=x^{(+)}+\frac{1}{a^{(+)}-v_{N}-v_{N P}-v_{P}} \cdot v_{N P} x^{(t)} .
$$

By substitution of Eq. (27) into Eq. (12); we obtain

$$
\begin{aligned}
& T_{f i}=\left(\Phi_{N}^{(-)}\left|v_{N P}\right| \varphi_{i}\right)+\left(\phi_{\mathbb{N}}^{(-)}\left|v_{P}\right| x^{(+)}\right) \\
& +\left(\phi_{N}^{(\omega)}\left|v_{P} \frac{1}{a^{(n)}-V_{N}-v_{N P}-v_{P}} v_{N P}\right| X^{(H)}\right):
\end{aligned}
$$

In a manner analogous to $\mathrm{Eq} .(27)$, we can define

$$
\psi^{(-)}=\Phi_{\mathbb{N}}^{(-)}+\frac{1}{(-)-V_{\mathbb{N}}-\mathbb{V}_{\mathbb{N P}}-\nabla_{P}} \cdot v_{P} \Phi_{\mathbb{N}}^{(-)}
$$

which reduces Eq. (28) to

$$
\mathrm{T}_{f i}=\left(\psi^{(-)}\left|v_{\mathbb{N P}}\right| x^{(+)}\right)+\left(\sigma_{\mathbb{N}}^{(\infty)}\left|v_{P}\right| x^{(+)}\right)+\left(\Phi_{N}^{(-)}\left|v_{N P}\right| \varphi_{i}-x^{(+)}\right) .
$$

Using Eqs. (26) and (10), we finally obtain

$$
\mathrm{T}_{f i}=\left(\psi^{(-)}\left|\mathrm{v}_{\mathbb{N P}}\right| x^{(+)}\right)+\left(\varphi_{i}\left|\mathrm{v}_{\mathrm{P}}\right| x^{(+)}\right)
$$

which is equal to

$$
\mathrm{T}_{f i}=\left(\psi^{(\omega)}\left|\mathrm{v}_{\mathrm{NP}}\right| x^{(+)}\right)+\left(x^{(-)}\left|\mathrm{v}_{\mathrm{P}}\right| \varphi_{1}\right)
$$

Equation (31) is the same as Eq. (4.4) of Gellomenn and Goldberger.*

The first term in Eq. (31) gives the transition amplitude for the pickup process occurring via the $V_{\mathrm{NP}}$ interaction. The incident proton interacts It should be noted that their Eq. (4.4) contains a misprint. In the first term, $\chi_{a}^{(t)}$ should read $\psi_{a}^{(t)}$. 
with all nucleons except the neutron to be picked up, and both the neutron and proton in the emerging deuteron interact with the residual core nucleons. The second term of Eq. (31) gives the amplitude for proton scattering from the nucleus via the interaction $V_{P}$ with just the $A-1$ nucleons of the core. This term, therefore, should not lead to final-state deuterons, since the deuteron binding potential $V_{N P}$ does not apperr in lle flualstate wave function, $x^{(-)}$. A formal proof that this term is indeed zero has been given by Lippmann, ${ }^{2} 7$ and 1 s obtained below in a manner similur to that described after Eq. (19) in Sertion 3. We first operate on the final state with $V_{P}$, and then let the results of the operation act on the initial state. The second term of Eq. (31) becomes

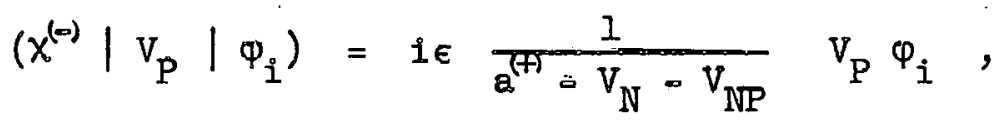

which can then be expanded in terms of $\phi_{k}$, the eigenfunctions of

$$
\begin{aligned}
\mathrm{H}_{0}+\mathrm{T}_{\mathrm{N}}+\mathrm{T}_{\mathrm{P}}+\mathrm{V}_{\mathrm{N}}+\mathrm{V}_{\mathrm{P}} \text {, to give } \\
\qquad i \in \int d E_{k} \sum_{k} \frac{\phi_{k}\left(\phi_{k}\left|\mathrm{v}_{\mathrm{P}}\right| \phi_{i}\right)}{\mathrm{E}_{i}-\mathrm{E}_{\mathrm{k}}+i \epsilon} .
\end{aligned}
$$

Because of the ie factor, the above expression is asymptotically zero unless $E_{1}=E_{k}$. However, the contribution to the integral from the one point $E_{i}=E_{k}$ will be vanishingly small unless the integrand contains a delta function in energy at this point. Because the initial and the final states of the neutron and proton are independent and uncoupled, their wave functions may be separately factored. Thus any delta function of the energy must be of the form $\delta\left(\mathrm{E}_{\mathrm{N}_{\mathrm{i}}}-\mathrm{E}_{\mathrm{N}_{\mathrm{k}}}\right) \delta\left(\mathrm{E}_{\mathrm{P}_{\mathrm{i}}}-\mathrm{E}_{\mathrm{P}_{\mathrm{k}}}\right)$. Therefore the second term of Eq. (31), vanishes asymptotically for our case in which the neutron changes from a bound state to an unbound 
state, since a delta function on the neutron energy cannot appear. We finally obtain

$$
\mathrm{T}_{f i}=\left(\psi^{(-)}\left|\mathrm{v}_{\mathrm{NP}}\right| x^{(+)}\right) \cdot
$$

We may now make our optical-model approximations (see the discussion following $\mathrm{Eq} \cdot(14))$ :

$$
x^{(+)} \simeq x_{C}^{(+)}=\varphi_{i}+\frac{i}{a^{(+)}-v_{N}} v_{C P} x_{C}^{(t)}
$$

and

$$
\psi^{(-)} \simeq \psi^{(-)}=\varphi_{\mathrm{C}}+\frac{1}{a^{(-)}-v_{\mathrm{N}}-v{ }_{\mathrm{CP}}-v t_{\mathrm{CN}}} \quad\left(\nu t_{\mathrm{CP}}+v \dagger_{\mathrm{CN}}\right) \varphi_{\mathrm{f}},
$$

which yield our desired symmetric expression,

$$
\mathrm{T}_{f i}=\left(\psi_{\mathrm{C}}^{(-)}\left|\mathrm{V}_{\mathrm{NP}}\right| x_{\mathrm{C}}^{(+)}\right)
$$

The differences between $\mathrm{Eq}$. (25) and $\mathrm{Eq}$. (35) are now apparent. The Initial state of the former describes a wave of incoming protons interacting with all $\mathrm{A}$ nucleons of the initial nucleus, while the latter represents interactions with only the A-l nucleons of the core. But, to compensate for this difference, the final-state wave function of Eq. (25) has only neutron interactions, whereas the corresponding wave function of Eq. (35) has both neutron and proton interactions. Therefore some of the proton interactions that seem to be missing in the initial state of Eq. (35) are included in its final state. Thus it is evident 
that within the optical-model approximations Eq. (35) is more exact and more aesthetically appealing than Eq. (25).

On the other hand, for practical reasons, Eq. (25) may in many cases offer a more suitable form for numerical calculations. This is true in the present case since 1t is desired to compare the opticalnodel theury wlih experimental rooulte for the reartion $\mathrm{r}^{12}(\mathrm{p}, \mathrm{d}) \mathrm{c}^{11}$. The optical parameters for $\mathrm{p}-\mathrm{C}^{12}$ scattering are certainly known better thän those fur $\mu-C^{11}$, which would have to be used in eva.7uating $E q .(35)$. Likewise, if one makes a final-state approximation that the deuteruil propagates as a single coherent particle, the optical parameter $U_{\mathrm{CN}}$ used in (25) is much better known than $v_{\mathrm{CD}^{2}}$ the deuteron optical potential, which would be required in (35). These arguments for the use of single-nucleon potentials are even more important when the potentials include spin-orbit effects:

One would then conclude that Eq. (35) is formally a more desira ahle form for the transition amplitude, but practical necessities may often favor the use of Eq. (25). 
II. EVALUATION OF THE MATRIX ELEMENT

$\therefore$ FOR CENIRAL POTENTTAL INTERACTIONS

\section{Section 1. Derivation of Optical-Model Wave Functions}

It was stated in the Introduction that two major problems arise in the attempt to accurately describe initial- and final-state scatterIng processes for a rearrangement collision such as the pickup process. The first task, which is that of finding an adequate formal approximation to the exact scatterlag, has been taken care of in Part I with the introduction of elasticascattering wave functions. The problem that remains is to find manageable forms of these wave functions so that the required integrations may be performed without excessive difficulty. In this section some explicit forms for the wave functions $\delta_{\delta^{(t)}} \mathrm{CP}_{i}$ and $\phi_{\delta^{(t)}} \mathrm{CN} \varphi_{f}$ are derived.

We have seen from $\mathrm{Eq} \cdot(14)$ of Part I that $\delta_{\delta^{(H)}} \mathrm{CP} \varphi_{i}$ satisfies an equation

$$
\left(\mathrm{H}_{\mathrm{O}}+\mathrm{T}_{\mathrm{P}}+\mathrm{T}_{\mathrm{N}}+\mathrm{V}_{\mathrm{N}}+V_{\mathrm{CP}}-\mathrm{E}_{i}\right) \Omega_{\mathrm{CP}}^{(+)} \Phi_{i}=0 .
$$

Separating the nuclear and proton coordinates as in Eq. (1) of Part I, we obtain an equation for the proton wave function,

$$
\left(-\frac{x^{2}}{2 m} \vec{\nabla}_{r_{P}}+V_{C P}\left(\vec{r}_{P}\right)-E_{P_{0}}\right) \psi_{0}\left(\vec{r}_{P}\right)=0 \text {, }
$$

where

$$
\Omega^{(+)}{ }_{C P} \varphi_{1}=x_{1}\left(\vec{r}_{A}\right) \Psi_{0}\left(\vec{r}_{P}\right)
$$

and

$$
\mathrm{E}_{\mathrm{P}}=\frac{\pi^{2} \cdot \mathrm{k}^{2} 0}{2 m}
$$


Now we can change Eq. (2) into a onemdimensional problem, and obtain a solution for $\Psi_{0}\left(\vec{r}_{\mathrm{P}}\right)$ in a closed-form WKB approximation. ${ }^{28}$ Equation (2) becomes

$$
\left(-\frac{x^{2}}{2 m} \frac{d^{2}}{d x^{2}}+v_{C P}(x)-E_{P_{0}}\right) \psi_{0}(x)=0 \text {, }
$$

which has as its Ifirstourder WKB solution;

$$
\psi_{0}(x) \sim \exp \left\{\frac{i}{x} \int \sqrt{2 m\left[E_{P_{0}}-V_{C P}(x)\right]} \cdot d x\right\} \text {. }
$$

We make the substitutions,

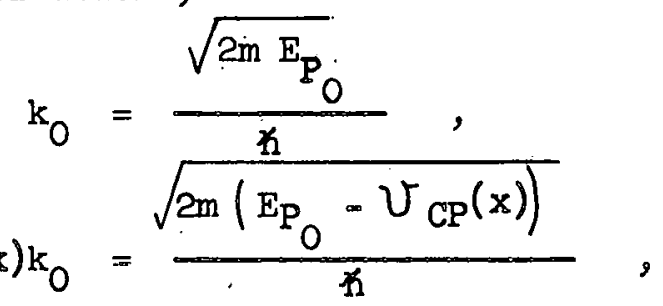

where

$$
n_{1}^{2}(x)=1-\frac{U_{\mathrm{CP}}(x)}{{ }_{\mathrm{E}_{0}}}
$$

defines the index of refraction in the nuclear medium. Equation (5) becomes

$$
\psi_{0}(x) \sim \exp \left\{i_{0} \int^{x} n_{l}(x) d x\right\} .
$$

To find the threedimensional form of $\psi\left(\vec{r}_{P}\right)$, we need to assume some distribution and shape for $\mathcal{V}_{C P}\left(\vec{r}_{P}\right)$. If we assume, for instance, a square well for a cylindrically shaped nucleus, then we may write, for $-R_{0}<x<R_{0} ; V_{C P}(x)=V_{0}$, and $n_{1}(x)=n_{1} ;$
for $x<-R_{0}$ and $x>R_{0} ; V_{C P}(x)=0$, and $n_{1}(x)=1$ These conditions allow us to integrate Eq. (8) to obtain 


$$
\begin{aligned}
\psi_{0}(x)= & \exp \left\{i k_{0} \int^{-R_{0}} d x+i i_{1} k_{0} \int_{-R_{0}}^{x} d x\right\} \\
& =\exp \left\{i\left(n_{1}-1\right) k_{0} R_{0}+i n_{1} k_{0} x\right\},
\end{aligned}
$$

which is a very simple closed form for the wave function in the region inside the nucleus. However, calculations that used such a cylindrical nucleus gave an angular distribution that was strongly peaked in the forward direction, in contrast to the broader angular distribution obtained from an exact numerical integration for a spherical nucleus. ${ }^{*}$ Except for order of magnitude calculations, therefore, the use of Eq. (8) should be restricted to the case of a spherical nucleus, for which we may write

for $r<R_{0} ; V_{C P}(\vec{r})=V_{0}$, and $n_{1}(\vec{x})=n_{1}$ for $r>R_{0} ; v_{C P}(\vec{r})=0, \quad$ and $n_{1}(\vec{r})=1$, which, for an incoming wave parallel to the $x$ axis, becomes

$$
\begin{array}{ll}
x<-\sqrt{R_{0}^{2}-r^{2} \sin ^{2} \theta}, & n_{1}(x)=1 \\
x>-\sqrt{R_{0}^{2}-r^{2} \sin ^{2} \theta}, & n_{1}(x)=n_{1}
\end{array}
$$

This may be easily seen by considering the scattering amplitude in Born approximation for a square-well potential. For the well-aknown spherical case, ${ }^{28}$. we have $f(\theta) \sim \frac{j_{1}\left(\Delta k R_{0}\right)}{\Delta k R_{0}}$, which has its first zero at $\Delta k R_{0} \simeq 4.5$. With a cylindrical shape, $f(\theta) \sim j_{0}\left(\Delta k R_{0}\right)$ where the first zero occurs at $\Delta \mathrm{kR}_{\mathrm{O}}=\pi$, and gives, therefore, a larger forward peak. 
Substituting $x=r \cos \theta$, we can evaluate Eq. (8) to obtain

$$
\psi_{0}(\vec{r})=\exp \left\{i k_{0} \int_{0}^{-\sqrt{R^{2}-r^{2} \sin ^{2} \theta}} d x+i n_{1} k_{0} \int_{-\sqrt{R^{2}-r^{2} \sin ^{2} \theta}}^{\cos \theta} d x\right\}
$$

or

$\psi_{0}(\vec{r})=\exp \left\{\ln _{1} \vec{k}_{0} \cdot \vec{r}+1\left(n_{1}-1\right) \vec{k}_{0} \sqrt{R_{0}^{2}-r^{2} \sin ^{2} \theta}\right\}$.

We note first that $\mathrm{Eq} .(10)$ obtains the classical result for a particle following a trajectory through a spherical region that is characterized by an index of refraction $n$. The fact that the classical expression is obtained is not surprising, since we have used the WKB solution of zero order in $\hbar$. Secondly, we see that Eq. (10) is more difficult to handle than the corresponding solution for a cylindrical nucleus. But by use of the approximation $r^{2} \sin ^{2} \theta<<R_{0}^{2}$, we arrive at an easily integrable expression,

$$
\psi_{0}(\vec{r}) \simeq \exp \left\{i\left(n_{1}-1\right) k_{0} R_{0}+i n_{1} \vec{k}_{0} \cdot \vec{r}\right\},
$$

which has the same form as the scattering wave functions of Hart and Montroll, 29 if one neglects the internal reflected wave. For $n \leqslant 0.10$, one obtains results from Eq. (11) that compare favorably to more exact numerical calculations that use Eq. (10). For larger values of $\mathrm{n}$, Eq. (10) may be approximated by

$$
\psi_{0}(\vec{r}) \simeq \exp \left\{\begin{array}{c}
\operatorname{in}_{1} \vec{k}_{0} \cdot \vec{r}+i\left(n_{1}-1\right) k_{0}\left[R_{0}-r(1-|\cos \theta|)\right] \\
\therefore
\end{array}\right\}
$$


which is, however, a less manageable function than that of Eq. (11). In the outside region, $r>R_{0}$, the wave function is merely $\exp \left(1 \vec{k}_{0} \circ \vec{r}\right)$. The small contribution of the transmitted wave has been neglected here.

We now turn to the problem of obtaining a form for the final. state wave function of Eq. (25). From Eq. (24) of Part I, we write the wave equation for $\Omega_{\mathrm{CN}}^{(\infty)} \varphi_{f}$, specifying the coordinates explicitly,

$$
\left(H_{0}\left(\vec{\tau}_{A-1}\right)+T_{R}(\vec{R})+T_{r}(\vec{r})+V_{N P}(\vec{r})+v_{C N}^{+}\left(\vec{r}_{N}\right)-E_{i f}\right) \delta_{C N}^{(\infty)} \varphi_{f}\left(\vec{r}, \vec{R}, \vec{\tau}_{A-I}\right)=0
$$

where $E_{\text {if }}=\frac{\pi^{2} K^{2}}{4 M}-B_{D}$, and $B_{D}$ is the magnitude of the deuteron binding energy. In order to solve Eq. (13), we assume $R>>r$, so that we have

$$
V_{\mathrm{CN}}^{t_{\mathrm{n}}}\left(\vec{r}_{\mathrm{n}}\right)=v_{\mathrm{CN}}^{\dagger}\left(\overrightarrow{\mathrm{R}}-\frac{\vec{r}}{2}\right) \simeq V t_{\mathrm{CN}}(\overrightarrow{\mathrm{R}})
$$

This means that we approximate the deuteron as behaving like a single coherent particle in the nucleus, or, more exactly, we assume that the average neutron-proton separation in the deuteron is small compared with the nuclear dimensions. A justification for this can be found in Appendix II.

Following our method for the proton wave function, we separate the nuclear and deuteron coordinates and obtain.

$$
\left(-\frac{x^{2}}{4 m} \nabla_{R}^{2}(\vec{R})-\frac{x^{2}}{m} \nabla_{r}(\vec{r})+v \dagger_{C N}(\vec{R})+V_{N P}(\vec{r})-E_{i f}\right) \phi_{D}(\vec{r}) x^{(-)}(\vec{R})=0 .
$$


We can further simplify Eq. (15) by subtracting

$$
\left(-\frac{\hbar^{2}}{m} \gamma_{r}(\vec{r})+v_{N P}(\vec{r})+B_{D}\right) \emptyset_{D}(\vec{r})=0,
$$

the equation for the bound state of the deuteron. We are left with

$$
\left[-\frac{x^{2}}{4 m} \cdot \gamma_{R}+v_{C N}-\left(E_{i f}-B_{D}\right)\right] x^{(-)}(\vec{R}) ;
$$

which can be solved by the WKB methud uutlined above. However, the form will not be quite the same as the result in Eq. (10), since. $88^{(-)} \mathrm{CN} \Phi_{f}$ or $x^{(-)}$is a solution that is asymptotic to outgoing plane waves at infinity and to incoming spherical waves. ${ }^{2}$ Therefore, its magnitude must be normalized to unity as it leaves the nucleus. $\left(x=R_{0}, \cos \theta>0\right)$, just as the magnitude of the incoming wave of $\mathrm{Eq} .(10)$ is unity as it enters the nucleus $\left(r=R_{0}\right.$, $\left.\cos \theta<0\right)$. For a spherically symetric square-well potential, we find

$x_{0}^{(-)}(\vec{R}) \sim \exp \left\{i^{\prime}{ }_{2} \vec{k} \cdot \vec{R}-i\left(n_{2}^{\prime}-1\right) K \sqrt{R_{0}^{2}-R^{2} \sin ^{2} \dot{\theta}}\right\}$,

where

$$
\left(n_{2}^{\prime}\right)^{2}=1-\frac{v_{C N}^{\dagger}}{E_{D}} \text {, }
$$

and

$$
E_{D}=\frac{\hbar^{2} K^{2}}{4 M}
$$

The approximations of Eqs. (11) and (12) may then be applied to Eq. (18) in order to obtain an easily integrable expression for the outgoing deuteron wave function.

It should be pointed out that, in the limit of small $n$, wave functions of the form of Eq. (II) can be handled with no more difficulty than the plane waves of the Born approximation. 


\section{Section 2. Evaluation of the Cross Section}

The wave functions of the preceding section may now be substituted into Eq. (25) of Part I to find the transition amplitude, $T_{f i}$, for the pickup process. The differential cross section is

$$
\frac{d \sigma}{d \Omega}=\sum_{f} \frac{3}{4} \frac{m^{2}}{3 \pi^{2} \pi^{4}} \frac{K}{k_{0}} \cdot \frac{A(A-I)}{(A+1)^{2}}\left|T_{f i}\right|^{2} .
$$

The factor of $3 / 4$ is due to spin statistics, and the sum is over all final states. The sum can be written

$\sum_{\vec{f}}\left|T_{f i}\right|^{2}=\sum_{f}\left|\left(x_{f}\left(\vec{\tau}_{A-1}\right) \phi_{D}(r) \psi_{0}^{(-)}(\vec{R})\left|v_{N P}(\vec{r})\right| x_{i}\left(\vec{\tau}_{A \infty 1}, \vec{r}_{N}\right) \psi_{0}^{(+)}\left(\vec{r}_{P}\right)\right)\right|^{2}$

or

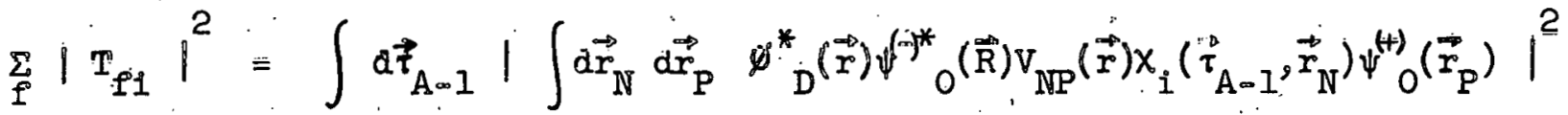

We need next to evaluate the integral,

$I=\int d \vec{r}_{N} d \vec{r}_{P} \phi^{*}(r) \psi^{(-)^{*}}(\vec{R}) v_{N P}(\vec{r}) x_{1}\left(\vec{r}_{A-I}, \vec{r}_{N}\right) \psi_{0}^{(H)}\left(\vec{r}_{P}\right)$

By a.llowing $v_{N P}(\vec{r})$ to operate on $\phi_{D_{i}}(\vec{r})$, we obtain $\left(\frac{\vec{r}^{2}}{m} \gamma^{2}-B_{D}\right) \phi_{D}(\vec{r})$, in accordance with Eq. (16). Therefore we denote

$$
V_{\mathrm{NP}}(\vec{r}) \emptyset_{\mathrm{D}}(\vec{r})=\varnothing(\vec{r})
$$

and define its Fourier transform:

$$
\phi(\vec{r})=\frac{1}{(2 \pi)^{3 / 2}} \int d \vec{k} \phi(\vec{k}) e^{i \vec{k} \cdot \vec{r}}
$$

Using Eqs. (25) and (18), we have 


$$
\begin{aligned}
I= & \left.\frac{e^{i\left(n^{\prime *} 2^{-1) k R}\right.}}{(2 \pi)^{3 / 2} \int d \vec{k} \phi^{*}(\vec{k})} \int d \vec{r}_{N} x_{i}\left(\vec{r}_{A-I^{\prime}} \vec{r}_{N}\right) e^{-i\left(\vec{k}+n^{\prime *}\right.} 2 \frac{\vec{K}}{2}\right) \cdot \vec{r}_{N} \\
& x \int d \vec{r}_{P} e^{i\left(\vec{k}-n_{2}^{\prime *} \frac{\vec{K}_{2}}{2} \cdot \vec{r}_{P}\right.} \psi_{0}\left(\vec{r}_{P}\right) .
\end{aligned}
$$

In $E_{1}$. (26) we have used the form of $\psi_{0}^{(0)}(\vec{R})$ inside the nuclear region where the putential $\mathcal{V}_{\mathrm{CN}}\left(\vec{r}_{N}\right) \simeq \mathcal{V J}_{\mathrm{CN}}(\vec{R})$ is effective. Contributions to the $r_{N}$ integral from the exterior region should be small, since the wave function $x_{i}\left(\vec{r}_{A \circ l}, \vec{r}_{N}\right)$ of the initial nucleus falls to zero rapidly at $r_{N} \simeq R_{0}$ (see Appendix II). At lower energies, where there is strong absorption in the nuclear medium, the contributions from the tail of the nuclear wave function probably could not be neglected. 6

The integration over $r_{N}$ now gives the partial Fourler transform of the initial wave function. After squaring and performing the integration over the ${ }^{\tau} \mathrm{A}-1$ coordinates of the core nucleons, we obtain the distribution of our initial-state neutrons in momentum space:

$$
\int \vec{d}_{A-1}\left|x_{i}\left(\vec{\tau}_{A-1}, \vec{n}\right)\right|^{2}=N(\vec{n})
$$

We now assume that we may use just the square root of this neutron momentum density distribution, $N(\vec{n})$, in our integral over $\vec{k}$. When we set

$$
[n(\vec{n})]^{1 / 2}=\Phi(\vec{n})
$$

Eq. (22) becomes

$$
\sum_{f}\left|T_{f i}\right|^{2}=|M|^{2}
$$


where

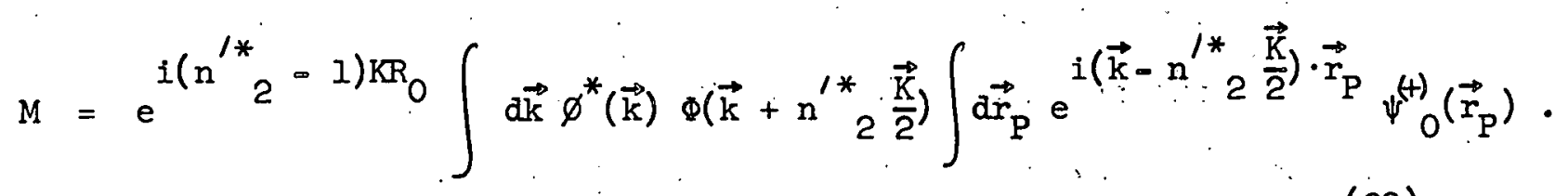

We may now perform the $r_{p}$ integration, using Eq. (11) for $r_{P}<R_{0}$, and $\psi_{O}^{(+)}\left(r_{P}\right)=e^{i \vec{k}_{0} \cdot \vec{r}_{P}}$ for $r_{P}>R_{0}$. Thus, we finally obtain our ex pression for the matrix element:

$$
\begin{gathered}
M=e^{i\left(n^{\prime *} 2-1\right) K R_{0}} \int d \vec{k} \phi^{*}(\vec{k}) \Phi\left(\vec{k}+n^{i *} 2 \frac{\vec{K}}{2}\right) \\
\times\left[\int_{r_{P}<R_{0}} d \vec{r}_{P} e^{i\left(n_{1}-1\right) k_{0} R_{0}+i\left(\vec{k}-n^{\prime *} 2 \frac{\vec{K}}{2}+n_{1} \vec{k}_{0}\right) \cdot \vec{r}_{P}}\right. \\
\left.\left.+\int_{r_{P}>R_{0}} d \vec{r}_{P} e^{i\left(\vec{k}-n^{\prime *}\right.} 2 \frac{\vec{K}}{2}+\vec{k}_{0}\right) \cdot \vec{r}_{P}\right]
\end{gathered}
$$

It should be pointed out here that Eq. (30) reduces to the Bornapproximation expression of the Chew-Goldberger theory for $n_{1}=n_{2}=1$. Since the incoming proton would then be represented by a plane wave, the integral over $r_{P}$ would give a delta function, $8\left(\vec{k}-\frac{\vec{k}}{2}+\vec{k}_{0}\right)$, which permits immediate evaluation of the integral.

One may gain some physical insight into the pickup problem, and, in fact, into the optical-model approach, from Eq. (30). If there were no optical potentials, i.e., $\mathrm{n}_{1}=\mathrm{n}_{2}=1$, the resulting delta function would require that, at the. instant of pickup, the relative momentum $\vec{k}$ 
be equal to $\frac{\vec{K}}{2}-\vec{k}_{0}$. However, if the proton is allowed to interact with the core, the delta function is no longer present, but is replaced by some distribution function (in brackets, above) for $\vec{k}$ in terms of $\vec{k}_{0}$ and $\vec{K}$. Thus at the moment of pickup, $\vec{k}$ may have values different from $\frac{\vec{K}}{2}-\vec{k}_{0}$, which results in a broadening of the theoretical angular dis tribution for outgoing deuterons. This point is discussed further Ia Part III.

Since some effort has been spent to obtain integrable wave functions for use in the matrix element, it. would obviously be desirable to continue further and use analytic expressions in Eq. (30) that allow its solution in closed form. To accomplish this, we assume first that $\Phi$ has a Gaussian form,

$$
\Phi\left(\vec{k}+n_{2}^{\prime *} \frac{\vec{K}}{2}\right)=c \exp \left[-c\left(\vec{k}+n^{\prime *} \frac{\vec{K}}{2}\right)^{2}\right] .
$$

If we wish to account for nuclear recoil, Eq. (31) becomes

$$
\Phi(\vec{k}, \vec{k})=c \exp \left[-c\left(q \vec{k}+p n_{2}^{\prime *} \frac{\vec{k}}{2}\right)^{2}\right],
$$

where

$$
q=\frac{A \cdot 1}{A} ; \quad p=\frac{A+1}{A}
$$

The factor containing the deuteron wave function may be obtained from Eqs. (24) and (25).. We assume a Hulthén deuteron wave function,

$$
\emptyset_{D}(\vec{r})=A \frac{e^{-\alpha r}-e^{-\beta r}}{r},
$$

where $\alpha^{2}=\frac{2 m \mathrm{~B}}{\hbar^{2}}$, and we have chosen $\beta=6.2 \alpha$. Then after a little algebra, we find 


$$
\phi(\vec{r})=A \frac{\hbar^{2}}{m}\left(\alpha^{2}-\beta^{2}\right) \cdot \frac{e^{-\beta r}}{r},
$$

and

$$
\varnothing(\vec{k})=\frac{4 \pi}{(2 \pi)^{3 / 2}} A \cdot \frac{\hbar^{2}}{m}\left(\alpha^{2}-\beta^{2}\right) \times \frac{1}{k^{2}+\beta^{2}} .
$$

For ease of integration, the functional dependence of Eq. (34) can be accurately expressed as a sum of Gaussians. In fact, we have

$\frac{1}{k^{2}+\beta^{2}} \simeq \frac{1}{\beta^{2}}\left[0.902 e^{-0.729 k^{2} / \beta^{2}}+0.0971 e^{-0.0313 k^{2} / \beta^{2}}\right]$.

Finally, the integrations over $r_{\mathrm{P}}$ give spherical Bessel functions, which also may be approximated by a sum of Gaussian functions. The first-order spherical Bessel function may be written

$\frac{j_{1}(x)}{x} \simeq \frac{1}{3}\left[e^{-0.111 x^{2}}-0.121 e^{-0.290(x-5.20)^{2}}+0.038 e^{-0.460(x-9.00)^{2}}\right]$,

which is accurate to $x \leqslant 10$. It turns out that at the energies under consideration, $j_{1}(x) / x$ is required only for values of $x$ less than 3 ; therefore the first term of Eq. (36) is sufficiently accurate.

The integral of Eq. (30) now becomes very simple if we substitute the approximations of Eqs. (31) through (36). Our matrix element will be the sum of several integrals of the form

$$
\int d \vec{k} e^{-b k^{2}-c\left(q \vec{k}-p n^{\prime *}\right.} 2{\left.\frac{\vec{k}}{2}\right)^{2}-a\left(n^{\prime *} 2 \frac{\vec{k}}{2}-\vec{k}-n_{1} \vec{k}_{0}\right)^{2}}_{i},
$$


which can quickly be reauced to a constart factor times an integral:

$$
\int d \vec{k} e^{-d k^{2}+2 \vec{k} \cdot \vec{Q}}=e^{a^{2} / d}\left(\frac{\pi}{d}\right)^{3 / 2},
$$

where the vector $\vec{Q} \quad$ is a linear combination of the incoming proton momentum $\vec{k}_{0}$ and the outgoing deuteron momentum $\vec{K}_{0}$. The actual values of $\vec{R}$ and $d$ in Eq. (37) cepend on the parameters $a, b$, and $c$ for the particular Gausstan considered. Then Eq. (37a) gives the form for each of these Grussin terms which, when sumed, constitute the solution of Eq. (30). Flnally, Eqs。 (28) and (21) allow the calculation of the differentiai cross section.

It is of course realized that other methods of integrating Eq. (30) give results that are at least, as accurate as the method described above. However, the use of Gaussian functions is particularly helpful in evaluating the polarlzation in Part IV. 


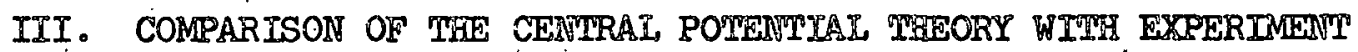
Measurements of the deuteron-pickup cross section bave been reported by Selove ${ }^{17}$ and by Coopers, 18 who beve obtalned angurar disw tributions for the process $\mathrm{C}^{12}\left(\mathrm{p}_{2} \mathrm{a}\right) \mathrm{C}^{1 \mathrm{l}}$, at 95 and 145 Mev respectively。 The results of the optical-model andysis are obtained by using the parameters listed in Table $I$, ad the resulting aragurar distributions are shown in Fis. I. The values or the araleora optleal potentials are taken Irom Glassgold. 30

Toble I

\begin{tabular}{|c|c|c|c|c|c|c|}
\hline \multicolumn{7}{|c|}{ Optical-model parameters for the process $c^{i 2}(p, d) c^{11}$. } \\
\hline $\begin{array}{l}F_{1} \text { 1ab } \\
\text { (Mev) }\end{array}$ & $\begin{array}{l}V_{\text {CF }} \\
\text { (Mer) }\end{array}$ & $\begin{array}{l}U_{\mathrm{CN}} \\
\text { (MeVi) }\end{array}$ & $n_{1}$ & $" u_{2}=m^{\circ} *^{2}$ & $R_{0} \times 10^{-13} \mathrm{~cm}$ & $\begin{array}{c}\Xi_{0} \\
(\text { Mev })\end{array}$ \\
\hline 95 & $-25-15 i$ & $-30-152$ & $1.130+0.0751$ & $1.175+0.0971$ & 3.4 & 20 \\
\hline 145 & $-25=15 i$ & $-20-158$ & $2.11+0.21120$ & $1.035+0.05641$ & 3.4 & 20 \\
\hline
\end{tabular}

It should be noticed from $\mathrm{Eq}$. (19) of Part II tagt $\mathrm{a}^{\circ *}=\mathrm{a}_{2}$ is $\mathrm{V}_{\mathrm{CN}}$ is in the form showa in Talle I above, i०e,g real and an imginary part. Mais follows irom the fact thet $\mathrm{a}_{2}{ }_{2}$ obtalned Prom $\mathrm{Vt}_{\mathrm{CN}}{ }^{*}$

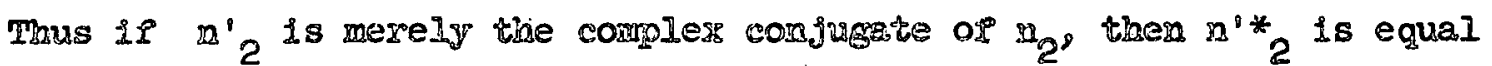
to $n_{2}$.

To obtain the results showin in Fig. 1 , the nuclear radius was chosen to be approximstely $1.5 \mathrm{~A}^{1 / 3}$ \& $10^{-13} \mathrm{~cm}_{1}$ and the melear momeratum distribution of the inftial nevticon state was of the form exp $\left(-\mathrm{E} / \mathrm{E}_{0}\right)$ 


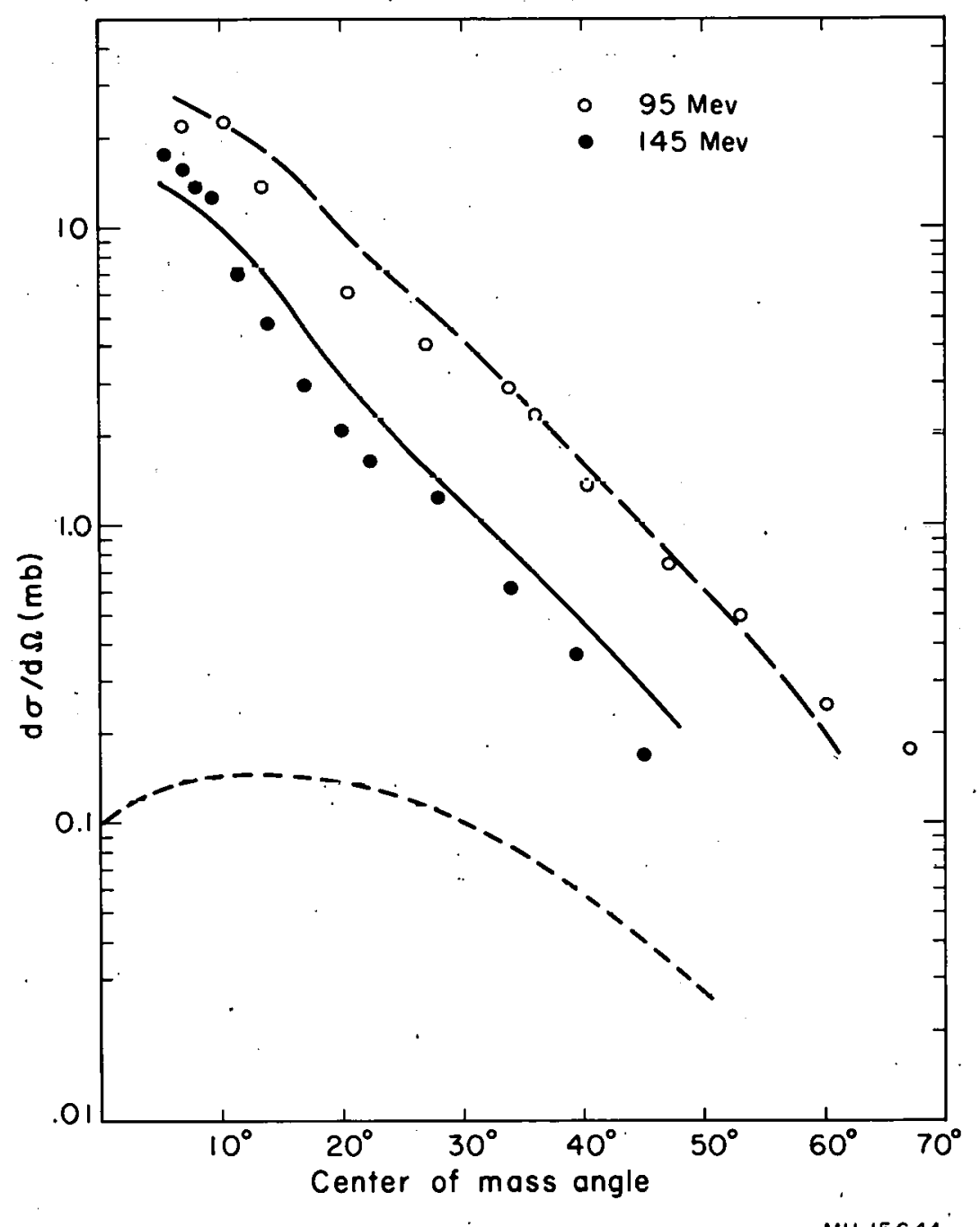

Fig. 1. The experimental and theoretical differential cross section for pickup at 95 and $145 \mathrm{Mev}$. The curve shown in dashes gives the approximate contributions to pickup from inelastic scatterings (see Appendix I). 
(see Eq. (3I) in Part II). Table I shows that the fit at both energies was obtained with $\mathrm{E}_{0}=20 \mathrm{Mev}$, which is in general agreement with the scattering results of Wilcox and Moyer, ${ }^{3 I}$ and Cladis, Hess, and Moyer. 32 Selove reported that in order to fit the Born approximation (Chew-Goldberger) theory to the results of his pickup experiments, he required high-momentum components in the nucleon momentum distribution, which had the form $\exp (-\mathrm{E} / 7)+0.15 \exp (-\mathrm{E} / 50) \cdot 17$ These high-momentum components $\left(E_{0}=50 \mathrm{Mev}\right)$ were needed to reproduce the observed wide-angle distribution of deuterons. In the Born-approximation theory, the deuteron momentum is just the sum of the incident proton momentum and the bound-state neutron momentum. In order to observe deuterons at appreciably large angles, therefore, one must have a nucleus that contains neutrons whose energy is about as large as the incident proton energy.

Such energetic neutrons are not required in the theory presented here, since the wide-angle distribution of deuterons can be obtained by allowing the incident and final particles to scatter in the nuclear field. For example, an incident proton may have scattered through a considerable angle with respect to the initial $\vec{k}_{0}$ direction before it encounters the neutron. Consequently, after the pickup of a low-energy neutron from inside the nucleus, a deuteron may emerge at an even larger angle. By further allowing this deuteron to scatter while leaving the nucleus, one can obtain very broad angular distributions.

If one integrates the neutron momentumødensity distribution over all momentum space, he obtains the number of neutrons that are effective in the pickup reaction. This number depends on the value of 
the constant $C$ in Eq. (31) of Part II, where $C$ is usually chosen so that the magnitude of the theoretical cross section fits the experimental results. In the fit to the experimental data shown in Fig. 1, seven neutrons were required at $95 \mathrm{Mev}$, and four neutrons at $145 \mathrm{Mev}$. These results, which approximately account for the six neutrons in $\mathrm{C}^{12}$, are in striking contrast to the 0.061 neutron obtained by seluve, and the 0.76 neutron obtained by Chew and Goldberger, in their Born-approximation analyses. The reason for the large differences is ot course due wo hie Inclusion of absorption effects in the sptical-model theory.

It should now be quite apparent that the neglect of the initialand final-state scattering contributions to the matrix element is not justified at these energies, if one is interested in finding accurate information concerning the detalls of nuclear structure. As one can see from the above discussion, the numerical results are often misleading. However, it should be pointed out that the Born approximation does predict the gruss features of the piokup prosess and gives reasonable angular distributions at small angles.

The different neutron numbers, four and seven, in the optical analysis are due to strong interference effects arising from the use of a square-well potential for the $V_{P}$ and $V_{N}$ interactions. It is believed that by utilizing a smother potential, such as the Saxon potential, one could fit both the $95-$ and $145-\mathrm{Mev}$ data with a single neutron number between four and six. In order to find a more exact number that would indicate whether pickup came from the four postate neutrons or all six neutrons, one would have to use more exact wave functions than those described in Eq. (11) of Part II. It would undoubtedly be necessary to 
use wave functions of the form of Eq. (10), Part II, and (or) higher orders in WKB approximation. In either case, a numerical integration of the matrix element would be inevitable.

Figure I shows that the theory reproduces some of the observed dip in the cross section at $20^{\circ}$. The failure to match this dip exactly is not an intrinsic fault of the theory, but is another consequence of the large interference cancellations that decrease the magnitude of the very-low-angle cross section (below $20^{\circ}$ ) (see discussion above). 
IV. EVALUATION OF THE. MATRIX ELEMENT: :

WITH SPIN-ORBIT POTENTIAIS INCLUDED

\section{Section 1. Derivetion of SpinoOrbit Optical-Model Wave Functions}

It is not necessary to change the general formalism of Part I in order to obtain infomation about the spin of the outgoing deuteron or about the spin effects in the differential cross section fur the pluku reaction. One need only assume a spin dependence as well as the. . spatial dependesce for the operators and rave functions used in the derivation of Equ. (25) and (35), and then apply the results of polaria zation formalism to extract the desired informetion. It can be seen from these two equations that spia effects in the pickup reaction may arise in three principal ways:

(a) The initialostate proton may become partially polarized while scattering from the auclear potential $\mathrm{V}_{\mathrm{P}}$.

(b) The main pickup interaction $v_{11 P}$ is a two body spin॰dependent potential, und way affect the apin orientation of the neutron and proton.

(c) The final-state devteron may become partialiy polarized while scattering in the residual mucleus.

In regard to the secord point above, Dalitz has shown that two spin effects of the $V_{N P}$ interaction can be neglected in Bornmapproximation treatments of the stripping (or pickup) resctions. 19 The first effect, that of producing tensor forces in the deuteron, is small, and the resulting Dostate deuterons contribute a negligible amount to the cross sections via the $V_{N P}$ interaction. The second effect of $V_{N P}$, that of causing deuteron polarization, is zero. This is because the proton spin is coupled only to the neutron spin, and polarization effects in the outgoing deuteron could occur only if there were a preferred 
direction for the spin of the neutron in the initial nucleus, which is not the case. Thus the Born-approximation theories must predict zero polarization for pickup deuterons. However, if one in addition considers the possibility of final-state deuteron absorption, he finds polarization effects from the $V_{N P}$ interaction. But such effects, considered by Malenka, ${ }^{24}$ are small and lead to polarizations whose maximum possible value is $1 / 3$.

All polarization effects of the $V_{N P}$ potential are neglected in the discussion that follows, as well as any spin-flip terms. It is therefore assumed that only the polarization from nuclear scattering is of major importance (points (a) and (c) above).

The polarization arising from nucleon-nudleus interactions can be ascribed to the presence of a spin-orbit term in each of the potentials $V_{C P}$ and $V_{C P N}$, which appear in the wave functions of Eqs. (25) and (35). Thus the desired description of any spin effects in the pickup reaction can be obtained by modifying the optical-model wave functions of Section I, Part II, to include these spin॰orbit terms. Such modifications are carried out in this section.

We may generalize Eq. (2) of Part II to read

$\left(-\frac{\hbar^{2}}{2 m} \gamma_{r_{P}}+V_{G P}\left(\vec{r}_{P}\right)+U_{C P}\left(\vec{r}_{P}\right) \vec{\sigma}_{P} \cdot \vec{b}_{P}-E_{P_{0}}\right) \psi\left(\vec{r}_{P}, \lambda_{P}\right)=0$,

where $U_{C P} \vec{\sigma}_{\mathrm{P}} \cdot \vec{l}_{\mathrm{P}}$ is the spin-orbit potential, $\vec{\sigma}$ gives the proton spin direction, and $\lambda_{\mathrm{P}}$ is the proton spin function. For $U_{\mathrm{CP}}\left(\vec{r}_{\mathrm{P}}\right)$ we use the notation of Fernbach, Heckrotte, and Lepore, 33 ..

$$
U_{C P}\left(\vec{r}_{p}\right)=\frac{\mu \dot{a}^{2}}{\hbar} \frac{1}{r} \frac{d}{d r} \rho(\vec{r}),
$$


where $\rho(\vec{r})$ gives the nuclear-density distribution. An approximate solution of $\mathrm{Eq}$. (1) can be obtained by considering the two-dimensional problem for waves moving parallel to the $x$ axis. Then we have

$$
\left[\frac{\hbar^{2}}{\partial m}\left(\frac{d^{2}}{d x^{2}}+\frac{d^{2}}{d y^{2}}\right)+V_{C P}(x)+U_{C P}(y) \vec{\sigma}_{C} \cdot \vec{y} \times \vec{p}_{x}-E_{P_{0}}\right] \psi(x, y)=0 .
$$

The spin-orbit potential, $U_{C P}$, is a function of the coordinate perpendicular to the motion of the particle. ${ }^{34}$ Although this two-dimensional model is a rather crude approximation, we may justify its validity by using it to solve the nucleon-nucleus scattering problem in. Born approximation. The resulting polarization is of the same form as the usual solution in spherical coordinates. In any case, the rigorous test for a solution of Eq. (3) is to see that it satisfies Eq. (1).

For a square-well density distribution, the spin-orbit potential becomes a delta. Iunction,

$$
U_{C P}(y) \vec{\sigma} \circ \vec{y} \times \vec{p}_{x}=\frac{\mu a^{2}}{\hbar} \frac{I}{y} \delta\left(y-R_{0}\right) \vec{\sigma}_{x} \cdot \vec{y} \times \vec{p}_{x} .
$$

Now, by assuming $\psi(x, y)$ separable into $X(x) Y(y)$, and anticipating that the approximate solution of $x(x)$ will be our WKB solution, $\Psi_{0}(x)$, of Eq. (1I), Part II, we can obtain $Y(y)$ in a straightforward manner with the help of Eq. (4). We find, after changing back to spherical coordinates,

$$
Y(\vec{r}) \simeq 1+\alpha \theta\left(r-R_{0}\right) \vec{\sigma} \cdot \vec{r} \times \vec{p}
$$


and

$$
\psi(\vec{r}) \simeq\left[1+\alpha \theta\left(r-R_{0}\right) \vec{\sigma} \cdot \vec{l}\right] \Psi_{0}(\vec{r})
$$

where $\alpha$ is a constant and $\theta\left(r-R_{0}\right)$ is a step function:

$$
\begin{array}{ll}
\theta\left(r-R_{0}\right)=1 ; & r<R_{0}, \\
\theta\left(r-R_{0}\right)=0 ; & r>R_{0} .
\end{array}
$$

In general we could replace the step function by a nuclear-density function $\rho(r)$ to obtain

$$
\psi(\vec{r})=[1+\alpha \rho(r) \vec{\sigma} \cdot \vec{l}] \Psi_{0}(\vec{r})
$$

The form of Eq. (5b) is made physically reasonable by considering Fermi's arguments. 35 Upon entering the nucleus, the particle feels two potentials: the central potential, which acts everywhere inside the nucleus and which changes the local wave number of the particle in accordance with the approximate WKB solution, and the spin-orbit potential, which acts only at the nuclear surface. Incoming particles feel a "kick" from this latter potential only while traversing the nuclear boundary, and they are not affected inside the boundary. If there is significant absorption of these particles in the nuclear medium, the "kick" they receive on their way out of the nucleus can be neglected in a first approximation. The step function or the density function of Eqs. (5) mathematically describes the physical arguments above.

To check the validity of Eq. (5b), and to find the exact value of the constant $\alpha$, we substitute the wave functions of Eq. ( $5 b$ ) into Eq. (1), and subtract Eq. (2) of Part II for the central potential. 
These steps leave us with

$$
\begin{aligned}
-\frac{\hbar^{2}}{2 m} \nabla^{2}[\alpha \rho(r) \vec{\sigma} \cdot \vec{l}] \Psi_{0}(r) & +[\beta \rho(r)-E] \alpha \rho(r) \vec{\sigma} \cdot \vec{l} \cdot \Psi_{0}(r) \\
& +r \rho^{\prime}(r) \vec{\sigma} \cdot \vec{l}[1+\alpha \rho(r) \vec{\sigma} \cdot \vec{l}] \Psi_{0}(r)=0,
\end{aligned}
$$

where we have used the general density function $\rho(r)$ rather than the specific step function, and

$$
\begin{aligned}
& V_{C}(r)=\beta \rho(r), \\
& U_{C}(r) \vec{\sigma} \cdot \vec{l}=r \rho !(r) \vec{\sigma} \cdot \vec{l} .
\end{aligned}
$$

Carrying out the indicated operations of Eq. (7) in spherical coordinates, we obtain the relation

$R_{0} \rho^{\prime \prime}\left(R_{0}\right)+4 \rho^{\prime}\left(R_{0}\right)+2$ in $k_{0} \cos \theta R_{0} \rho^{\prime}\left(R_{0}\right)+\frac{2 m}{\hbar^{2}} \frac{r}{\alpha} \rho^{\prime}\left(R_{0}\right)=0$,

where it has been assumed only that $\rho(r)$ falls to zero in a distance $2 \epsilon$ where $\epsilon \ll R_{0}$, and $\rho\left(R_{0}\right)=\frac{1}{2} \rho(0)$. If we average the quantities of $\mathrm{Eq}$. (9) over the falloff distance, $i_{\circ} e_{0}$, from $R_{0}-\epsilon$ to $R_{0}+\epsilon$, the first term is approximately zero. Then we are left with an equation that can be solved for $\bar{\alpha}$ :

$$
\bar{\alpha}=\frac{2 m \bar{\gamma}}{\hbar^{2}}\left[\frac{1}{4+2 \text { in } k_{0} R_{0} \cos \theta}\right] \text {. }
$$

In the limit of small $k_{0} R_{0}$ the second term of the denominator may of course be neglected." For larger $k_{0} R_{0}$ "it still may be neglected, since the main spin-orbit contributions arise in the region where $\theta$ is on 
the order of $\frac{\pi}{2}$. Therefore; from Eqs. (2) and (8); we obtain

$$
\bar{\alpha} \simeq \frac{2 m}{\hbar^{2}} \frac{\bar{r}}{4}=\frac{m \mu a^{2}}{2 \hbar^{3}} \text {. }
$$

We may use Eq. (II) of Part II to obtain the approximate solution of Eq. (i) for a square-well potential:

$\psi(\vec{r})=\left[1+\frac{m \mu a^{2}}{2 \hbar^{3}} \quad \theta\left(r-R_{0}\right) \vec{\sigma} \cdot \vec{l}\right] \cdot e^{i(n-1) k_{0} R_{0}+i n \vec{k}_{0} \cdot \vec{r}}$

For further verffication of our spin-orbit wave functions, one may use them to solve the polarization from the elastic scattering of nucleons by nuclei. The results, which can be obtained in closed form, are in excellent agreemint with experiment and with longer, more exact theoretical calculations. 33 


\section{Section 2. Derivation of the Spin Matrix}

The transition matrix of Eq. (25) in Part I describes the amplitude of the scattered wave in terms of the incoming wave. If the wave functions have a spin dependence, then the transition matrix also specifies the amplitude of the outgoing spin in terms of the incoming spin state, $\lambda_{P^{\circ}}$ From invariance argiments, it cun le shomn 25 that for two spin-l/? particles and (we assume) a spin-zero nucleus, the most general form of a matrix $M$ is

$$
M=A+B \vec{\sigma}_{\Gamma} \cdot \vec{n}+C \vec{\sigma}_{N} \cdot \vec{n}+\ldots,
$$

where

$$
\overrightarrow{\mathrm{K}} \times \overrightarrow{\mathrm{k}}_{0}=\overrightarrow{\mathrm{n}} \mathrm{K} \mathrm{k}_{0} \sin \theta
$$

is a vector normal to the plane of.scattering. For our purposes, the higher-order terms in Eq. (13) that involve products of $\sigma_{\mathrm{P}}$ and $\sigma_{\mathrm{N}}$ have been neglected to a first approximation, since in our elastic wave functions, the coefficients of $\sigma_{P}$ and $\sigma_{N N}$ are small.

If we now insert the spinworbit wave functions of $\mathrm{Eq}$. (12) for both the Initial and final states of the transition matrix (Eq. (25), Part I), we can evaluate the functions $A, B$, and $C$, which are used in the next section to find the polarization. The term in both wave functions that does not involve the spin leads to the matrix element. of Eq. (29), Part II, and we designate it $A(\theta)$ 。 To evaluate $B(\theta)$, we use the spin-dependent part of the proton wave function, and the spin-independent part of the deuteron wave function. Then we have 


$$
\begin{aligned}
& \vec{\sigma}_{P} \cdot \vec{n} B(\theta)=e^{i\left(n_{2}-1\right) K R_{0}} \int d \vec{k} \phi^{*}(k) \Phi\left(\vec{k}+n_{2} \frac{\vec{K}}{2}\right) \\
& \quad \times \quad \int_{r_{P}<R_{0}} d \vec{r}_{P} e^{i\left(\vec{k}-n_{2} \frac{\vec{K}}{2}\right) \cdot \vec{r}_{P}} \alpha_{P} \theta\left(r_{P}-R_{0}\right) \vec{\sigma}_{P} \cdot \vec{l}_{P} e^{i\left(n_{1}-1\right) k_{0} R_{0}+i n_{1} \vec{k}_{0} \cdot \vec{r}_{P}}
\end{aligned}
$$

The integral over $r_{p}$ is

$$
I_{P}=\hbar \alpha_{P} e^{i\left(n_{1}-1\right) k_{0} R_{0}} n_{1} \vec{k}_{0} \cdot \vec{\sigma}_{P} \times \int d \vec{r}_{P} \vec{r}_{P} e^{i\left(\vec{k}-n_{2} \frac{\vec{k}}{2}+n_{1} \vec{k}_{0}\right) \cdot \vec{r}_{P}} \Theta\left(r_{P}-R_{0}\right) .
$$

We set

$$
\vec{r}_{P} \theta\left(r_{P}-R_{0}\right)=\vec{\nabla}_{r_{P}} F\left(\vec{r}_{P}\right),
$$

and find

$$
F\left(\vec{r}_{P}\right)=\frac{r_{P}^{2}-R_{0}^{2}}{2} \theta\left(r_{P}-R_{0}\right) \text {. }
$$

Then Eq. (16) becomes

$$
\begin{aligned}
& I_{P}=\hbar \alpha_{P} e^{i\left(n_{I}-1\right) k_{0} R_{0}}{ }_{i} \vec{\sigma}_{P} \cdot \vec{k}_{0} \times\left(\vec{k}-n_{2} \frac{\vec{k}}{2}+n_{1} \vec{k}_{0}\right) \\
& \quad \int d \vec{r}_{P} \frac{r_{P}^{2}-R_{0}^{2}}{2} \theta\left(r_{P}-R_{0}\right) e^{i\left(\vec{k}-n_{2} \frac{\vec{K}}{2}+n_{1} \cdot \vec{k}_{0}\right) \cdot \vec{r}_{P}} .
\end{aligned}
$$

The $r_{p}$ integral of $\mathrm{Eq} \cdot(18)$ gives $-4 \pi, \mathrm{R}_{0}^{5} \frac{j_{2}\left(k R_{0}\right)}{\left(k R_{0}\right)^{2}}$; 
where $\vec{k}=\vec{k}-n_{2} \frac{\vec{k}}{2}+n_{1} \vec{k}_{0} \cdot$ The term in Eq. (18) involving $\vec{k}_{0} \times \vec{k}_{0}$ is of course zero. The $\vec{k}_{0} \times \vec{K}$ term may be taken out as a factor multiplying the $\overrightarrow{d \vec{k}}$ integration, which may be carried out by the method of Part II. However, the term involving $\overrightarrow{\mathrm{k}}_{\mathrm{O}} \times \overrightarrow{\mathrm{k}}$ must remain in the integrand of the integral over $\vec{k}_{\text {. }}$ This integral will have a form similar to Eq. (37a) of Part II, except for an addlllunal factor of $\overrightarrow{\mathbf{k}}$ :

$$
g=\int \overrightarrow{d \vec{k}} \vec{k} \cdot e^{-d k^{2}+2 \vec{k} \cdot \vec{R}}
$$

To obtain Eq. (19) we have made a Gaussian approximation for $\frac{j_{2}(x)}{x^{2}}$ similar to Eq. (36) of Part II. By setting

$$
\vec{k} e^{-d k^{2}}=\vec{\nabla}_{k} F(\vec{k}) \text {, }
$$

where

$$
F(\vec{k})=-\frac{1}{2 d} e^{-d k^{2}}
$$

we ubtain

$$
\begin{aligned}
\partial & =\frac{1}{2 \bar{d}} \int d \vec{k} \vec{\nabla}_{k}\left(e^{2 \vec{k} \cdot \vec{R}}\right) e^{-d k^{2}}=\frac{\vec{R}}{\vec{d}} \int e^{-d k^{2}+2 \vec{k} \cdot \vec{R}} \\
& =\frac{\vec{R}}{d} e^{Q^{2} / d}\left(\frac{\pi}{d}\right)^{3 / 2}
\end{aligned}
$$

where $\vec{R}$ is some linear combination of $\vec{K}$ and $\vec{k}_{0}$, as explained in Part II. Thus the proton-spin term of Eq. (15) can be obtained from Eqs. (16) through (2l)。 To obtain the neutron ispin term, $C(\theta)$, we use the spin-dependent part of the outgoing wave function, and the spinindependent part of the proton wave function. The calculation is similar 
to that of $B(\theta)$, except that, instead of the step-function distribution in the $\vec{r}_{\mathrm{P}}$ integration of Eq. (18), we have the product of a Gaussian distribution and a step function for the $\vec{r}_{N}$ integrand. (This arises from the use of a Gaussian form for the neutron-momentim distribution.) If the Gaussian falls to zero at $r_{N}=R_{0}$ (see Appendix II), then the product leaves us with just the Gaussian. Therefore instead of Eq. (17) we have

$$
x_{i}\left(\vec{r}_{N}\right) \vec{r}_{N}=C^{\prime} e^{-r_{N}^{2} / 4 c} \vec{r}_{N}=\vec{\nabla}_{r_{N N}} G\left(\vec{r}_{N}\right) \text {, }
$$

and find

$$
G\left(\vec{r}_{N}\right)=-2 c x_{i}\left(\vec{r}_{N}\right)
$$

Referring to Eq. (26) of Part II, we see that the $\sigma_{\mathrm{N}}$ matrix element is

$$
\begin{gathered}
c(\theta) \vec{\sigma}_{N} \cdot \vec{n}=\frac{e^{i\left(n_{2}-1\right) k R_{0}}}{(2 \pi)^{3 / 2}} \cdot \int d \vec{k} \phi^{*}(k) \int d \vec{r}_{P} e^{i\left(\vec{k}-n_{2} \frac{\vec{k}}{2}\right) \cdot \vec{r}_{P}} \psi_{0}\left(\vec{r}_{P}\right) \\
x \int d \vec{r}_{N} e^{-i \vec{k} \cdot \vec{r}_{N N}} \alpha_{N} \vec{\sigma}_{N} \cdot \vec{l}_{N} e^{-i n_{2} \frac{\vec{k}}{2} \cdot \vec{r}_{N}} x_{i}\left(\vec{r}_{A-1^{\prime}}, \vec{r}_{N}\right)
\end{gathered}
$$

In Eq. (24), the operator $\vec{b}_{N}$ operates on both the exponential and the neutron wave function $x_{i}\left(\vec{\tau}_{A-I}, \vec{r}_{N}\right)$. However, this operator commutes with $x_{i}$, if $x_{i}$ has the Gaussian form of Eq. (23). (Operating on the Gaussian with $\vec{r}_{N} \times \frac{1}{r_{N}} \frac{d}{d r} \vec{r}_{N}$ gives $\vec{r}_{N} \times \vec{r}_{N}=0$. ). Using Eqs. (22) and $(24)$, one finds 


$$
\begin{aligned}
& c(\theta) \vec{\sigma}_{N} \cdot \vec{n}=c \alpha_{N} \hbar e^{i\left(n_{2}-I\right) K R_{0}} n_{2} \vec{K} \cdot \vec{\sigma} \quad x \int d \vec{k}\left(\vec{k}+n_{2} \frac{\vec{K}}{2}\right): \phi^{*}(\vec{k}) \Phi\left(\vec{k}+n_{2} \frac{\vec{K}}{2}\right) \\
& x \quad \int d \vec{r}_{p} e^{i\left(\vec{k}-n_{2} \frac{\vec{K}}{2}\right) \cdot \vec{r}_{p}} \quad \therefore \quad \Psi_{0}\left(\vec{r}_{p}\right)
\end{aligned}
$$

The $\overrightarrow{\mathrm{K}} \times \overrightarrow{\mathrm{K}}$ term is zero, and the $\overrightarrow{\mathrm{K}} \times \overrightarrow{\mathrm{K}}$ term is handled in cractly the same way as described in Eqs. (19) through (21). Therefore the neutronspin term is obtained from Eqs. (22) through (25). The -product functions of $\sigma_{\mathrm{N}}$ and $\sigma_{\mathrm{P}}$ have been calculated, and their coefficients were found to be on the order of $1 / 100$ the magnitude of $B(\theta)$. Thus we may justifiably assume the spin matrix to have the form of Eq. (13). 


\section{Section 3. Analysis of the Polarization}

In the evaluation of the polarization of deuterons from the pickup reaction it is convenient to use the M-matrix notation of Wolfenstein and Ashkin. 25 Specific applications of this formalism to deuteron problems have been investigated by Lakin 36 and by Stapp. 37 However, since the tensor terms in the deuteron formalism turn out to be small for the pickup reaction, it is simpler for the purposes here to consider just the formalism for two spin-1/2 particles, and assume that the nucleus (or core) has zero spin. At the end, the desired deuteron states can be obtained by use of a projection operator.

We begin by developing a method to find the polarization states of outgoing particles in terms of the initial polarization state. Following Wolfenstein and Ashkin, the density matrix for a mixture of states is

$$
p_{\alpha \beta}=a_{\alpha} a_{\beta}^{\dagger}
$$

where $a_{\alpha}$ is a four component column vector representing the spin states of the two particles, and $a_{\alpha}^{\dagger}$ is its adjoint row vector. We next introduce sixteen Hermitian operators in the spin space, $s^{\mu}$, that form a complete set, i.e.,

$$
\operatorname{Tr}\left(\dot{S}^{\mu} S^{\nu}\right)=4 \delta_{\mu \nu}
$$

The $s^{\mu}$ are $4 \times 4$ matrices that may be combined from products of the unit matrix and the Pauli spin matrices:

$$
I_{\mathrm{P}} I_{\mathrm{N}} ; I_{\mathrm{P}} \sigma_{\mathrm{N}_{i}} ; \sigma_{\mathrm{P}_{i}} I_{\mathrm{N}} ; \sigma_{\mathrm{P}_{1}} \sigma_{\mathrm{N}_{j}}
$$

where $i$ and $j$ each take on the three values $x, y$, and $z$, and we 
designate the spin states of the neutron and proton separately. The average value of any spin operator $\mathrm{s}^{\mu}$, is given by

$$
<\mathrm{S}>\operatorname{Tr}(\ddot{\rho})=\operatorname{Tr}(\rho \mathrm{S})
$$

Since $\rho$ is a $4 \times 4$ matrix, it must be linearly expressible in the sixteen base wullices $0^{\mu}$, or, ueing $\mathrm{E}_{q}$ ( (P9),

$$
\rho=\frac{1}{4} \operatorname{Tr}(\rho) \sum_{\mu}<s^{\mu}>s^{\mu}
$$

The matrix that transforms the initial spin state into lie final atate is $M$ :

$$
a_{\alpha}=\sum_{\beta} M_{\alpha \beta} a_{\beta}:
$$

For spinless particles, $M$ is just the usual scattering amplitude (Eq. (29), Part II), but in general $M$ is a linear combination of the sixteen base matrices. The form we use in our particular case will be seen presently. From Egss. (31) and (26) we find an expression for the final density matrix,

$$
\rho_{f}=M \rho_{i} M^{\dagger} \ldots
$$

Using Eqs. (26) through (32), we find the desired relation for the averages $\left\langle\mathrm{s}^{\mu}\right\rangle_{f}$ of the final states in terms of the $\left\langle\mathrm{s}^{\mu}\right\rangle_{1}$ for the initial state

$$
\left\langle S^{\mu}\right\rangle_{f} I=\frac{1}{4} \sum_{j}\left\langle S^{\nu}\right\rangle_{i} \operatorname{Tr}\left(M S^{\nu} M^{\dagger} S_{!}^{\mu}\right)
$$

where

$$
I=\frac{\operatorname{Tr}\left(\rho_{f}\right)}{\operatorname{Tr}\left(\rho_{i}\right)}
$$

is the differential cross section. 
To clarify the form of these matrices, we may define the a's

$$
a=\left(\begin{array}{l}
a_{1} \\
a_{2} \\
a_{3} \\
a_{4}
\end{array}\right)
$$

where

$a_{1}=1$; proton spin up, neutron spin up:

$a_{2}=1$; proton spin up, neutron spin down;

$a_{3}=1$; proton spin down, neutron spin up;

$a_{4}=1 ;$ proton spin down, neutron spị down.

If the $z$ axis is defined to be along the normal to the plane of scattering, the M matrix of Eq. (13) becomes

$$
M=A(\Theta) I_{P} I_{N}+B(\theta) \sigma_{P_{2}} I_{N}+C(\theta) I_{P} \sigma_{N_{Z}},
$$

or, using the definition of Eqs. (35),

$M=A(\theta)\left(\begin{array}{cccc}1 & & & 0 \\ & 1 & & \\ 0 & & 1 & \\ 0 & & & -1\end{array}\right)+B(\theta)\left(\begin{array}{rrrr}1 & & & 0 \\ & 1 & & \\ 0 & -1 & -1 & \\ 0 & & -1\end{array}\right)+C(\theta)\left(\begin{array}{rrr}1 & & 0 \\ 0 & -1\end{array}\right)$.

Now we are in a position to investigate speciail cases of Eq. (33). For the pickup process, there are only four $\left\langle\mathrm{s}^{\nu}\right\rangle_{i}$ different: from zero; since the neutron to be picked up is unpolarized and can therefore be represented by the unit matrix. One case of interest is the 
polarization of deuterons produced by the scattering of unpolarized protons. For this case, all the $\left\langle s^{\nu}\right\rangle_{i}$ vanish except the unit matrix, and Eq. (33) becomes

$$
\left\langle S^{\mu}\right\rangle_{f} I=\frac{1}{4} \operatorname{Tr}\left(M M^{\dagger} S^{\mu}\right)
$$

The second interesting case is to find the acymmetry of deuterons resulting from the scattering of initially polarized protons. Here we find

$$
I=\frac{1}{4} \operatorname{Tr}\left[T\left(M M^{\dagger}\right)\right]+\frac{1}{4} \overrightarrow{P \mu} \cdot \operatorname{Tr}\left[T\left(M \vec{\sigma}_{P} I_{N} M^{\dagger}\right)\right],
$$

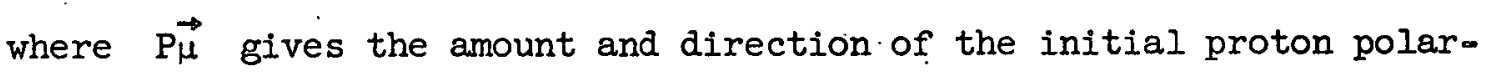
ization and a projection operator has been applied to Eq. (39) before the trace has been taken. This operator, $\mathrm{T}$, will project out the singlet states of the n-p system and leave only triplet deuteron states, 38

$$
T=\frac{3+\vec{\sigma}_{P} \cdot \vec{\sigma}_{N}}{4}
$$

The differential cross section then has the form

$$
I=I_{0}+I_{P},
$$

where $I_{0}$, the first term of Eq. (39), is the cross section for an unpolarized beam, and $I_{P}$, the second term, is the contribution from the initial proton polarization.

To illustrate a particular case, we assume that the initial polarization is in the $\mathrm{z}$ (or $\overrightarrow{\mathrm{n}}$ ) direction. Then the only nonzero $\left\langle S^{\mu}\right\rangle_{i}$ of Eq. (33) are the unit matrix: $l_{\mathrm{P}}: l_{N}$, and $: \sigma_{\mathrm{P}_{2}} l_{\mathrm{N}^{*}}$ or

$$
\rho_{i}=I_{P} I_{N}+P \rho_{P_{2}} I_{N} \text {. }
$$


Eq. (39) becomes

$$
I=\frac{1}{4} \cdot \operatorname{Tr}\left[\mathrm{T}\left(\mathrm{M} \mathrm{M}^{\dagger}\right)\right]+\frac{1}{4} \mathrm{P} \operatorname{Tr}\left[\mathrm{T}\left(\mathrm{M} \sigma_{\mathrm{P}} \quad \mathrm{T}_{\mathrm{N}} \mathrm{M}^{\dagger}\right)\right]
$$

With some relations of our $4 \times 4 \mathrm{~S}^{\mu}$ matrices,

$$
\begin{aligned}
& \operatorname{Tr}\left(\begin{array}{ll}
\sigma_{\mathrm{P}} & \sigma_{\mathrm{P}}
\end{array}\right)=\operatorname{Tr}\left(\sigma_{\mathrm{N}} \quad \sigma_{\mathrm{z}}\right)=4, \\
& \operatorname{Tr}\left(\sigma_{\mathrm{P}_{\mathrm{z}}} \quad \sigma_{\mathrm{N}}\right)=\operatorname{Tr}\left(\sigma_{\mathrm{P}}\right)=\operatorname{Tr}\left(\sigma_{\mathrm{N}}\right)=0,
\end{aligned}
$$

we can easily obtain, using Eqs. (44), (40), and (36),

$I=\frac{3}{4}\left(|A|^{2}+|B|^{2}+|C|^{2}\right)+\frac{1}{2} \operatorname{Re}\left(B C^{*}\right)+P\left\{\operatorname{Re}\left[A\left(\frac{3}{2} B^{*}+\frac{1}{2} C^{*}\right)\right]\right\}$.

The asymmetry is given by

$$
\epsilon=\frac{I_{L}-I_{R}}{I_{L}+I_{R}}
$$

where $I_{L}$ and $I_{R}$ are the differential cross sections to the left and right respectively. Since both $\mathrm{B}$ and $\mathrm{C}$ have $\mathrm{a} \sin \theta$ dependence,

$$
\epsilon=\frac{\operatorname{Re}\left[A\left(\frac{3}{2} B^{*}+\frac{i}{2} C^{*}\right)\right]}{\frac{3}{4}\left(|A|^{2}+|B|^{2}+|C|^{2}\right)+\frac{1}{2} \operatorname{Re}\left(B C^{*}\right)}
$$

is the expression for the asymmetry in the plane of scattering perpendicular to the direction of polarization. The leftaright asymmetry in directions other than the one specified above may be easily found from Eq: (39) without the restriction of Eq: (42). 


\section{COMPARISON OF THE DEUTERON ASYMMETRY WITH EXPERIMENT}

Experimental results for the left-right asymmetry have been recently reported by Cooper. ${ }^{18}$. To compare the optical-model spindependent theory with these data, the calculational methods of Part II, and the spin-dependent terms $B(-\theta)$ and $C(\theta)$. of Part IV, Section 2, are used. In addition to the purameleis liatcd in Tabjo I, the strength of the spin-orbit potential. (see Eqs. (8) and (1i) of Part IV) must be specified. These calculations have used lije value $\mu a^{2}=5 \times 10^{-26} \mathrm{Mev} \mathrm{\textrm {sm } ^ { 2 }}$ chocen by Fernharh, Heckrotte, and Lepore. 33 The results for the asymmetry of deuterons produced from $\mathrm{C}^{12}$ targets by 145-Mev polarized protons are shown in Fig. 2.

One can see that the theory does not completely reproduce the observed low asymmetry at angles below $15^{\circ}$. An explanation for this follows the arguments given in Part III. The use of square-well potentials in the theory gives rise to large cancellation in the spin-independent term $\mathrm{A}(\Theta)$ at angles below $15^{\circ}$. These cancellations are due to the interference of proton waves in the region $r_{P}>R_{0}$ with those inside the nucleus, $r_{P}<R_{0}$. Qualitatively, the use of a more smoothly varying potential would increase the small-angle contributions to $A(\theta)$, which would in turn produce more of a dip in the differential cross section at $20^{\circ}$, and also would lower the asymmetry for angles less than $15^{\circ}$ to $20^{\circ}$. However, the main features of the experimental data, particularly the large observed asymmetry, are predicted by the theory. From the arguments given at the beginning of Part IV, one can see that the major polarization effects indeed must come from the spinoorbit scatterings, since the results from considerations of the $\mathrm{v}_{\mathrm{NP}}$ inter- 


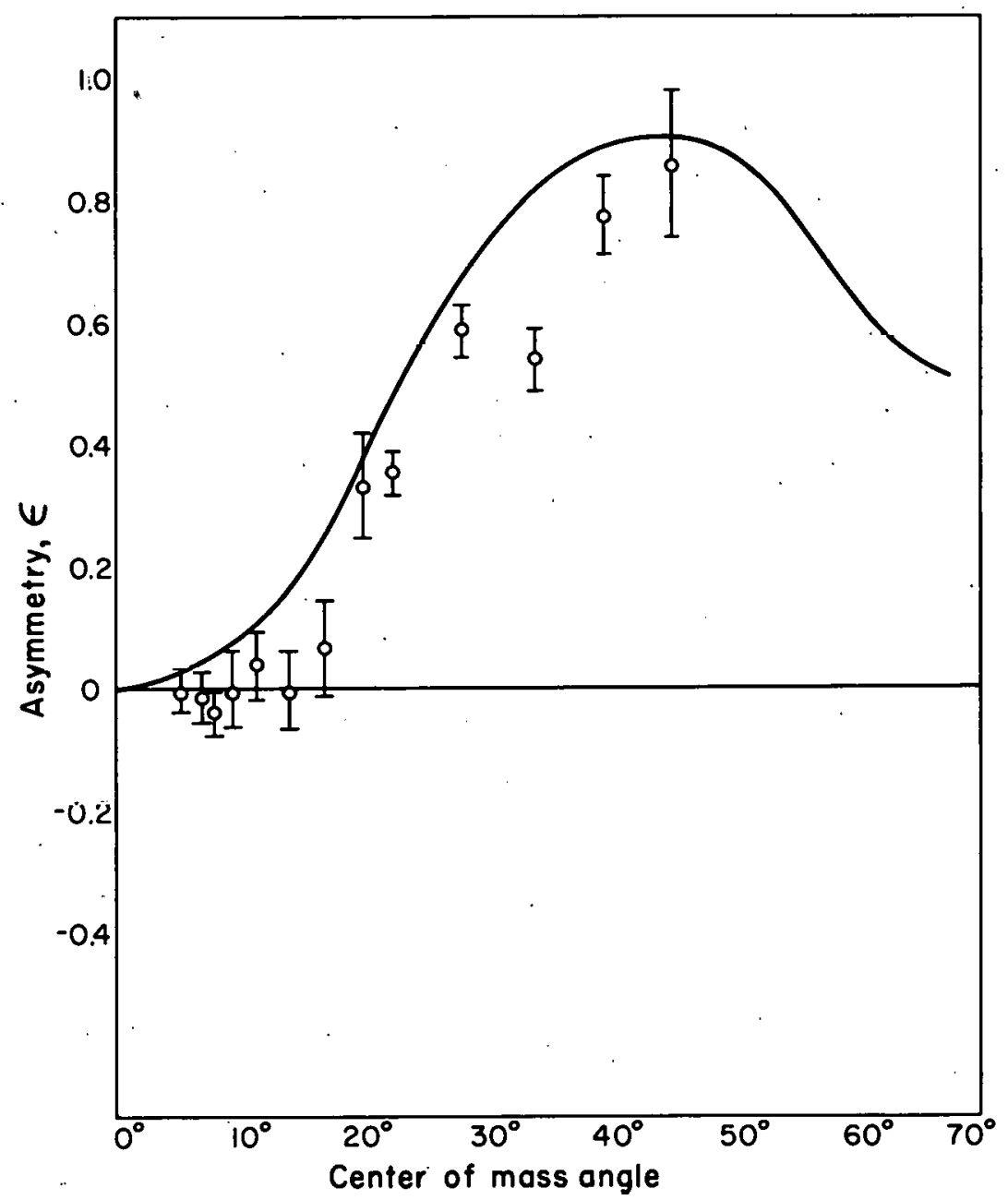

MU-15643

Fig. 2. The experimental and theoretical deuteron asymmetry obtained from pickup by 145-Mev polarized protons whose direction of polarization is perpendicular to the plane of scattering. 
$-60-$

action, as treated by Malenka, are much too small in magnitude to account for the observed asymmetry. 


\section{ACKNOWLEDGMENTS}

The author is deeply indebted to Professor Kenneth M. Watson, and to $\mathrm{Dr}$. Warren Heckrotte, who suggested this problem, for their sympathetic understanding, guidance, and continuing support throughout the course of this work:

Many other people have been helpful during the period of this research, including members of the staffs of the Radiation Laboratory and the Department of Physics at the University of California. Partico ular thanks are due to Drs. Henry Stapp and Leonard Rodberg for many stimulating discussions and helpful suggestions.

This work was performed under the auspices of the United States Atomic Energy Commission. 


\section{APPENDIX I. INELASTIC -SCATTERING CONIRIBUTIONS}

Various qualitative arguments that the inelastic-scattering corrections to the cross section are small were made in Part $I$, and in the development of the opticalmodel wave functions in Part II. . A quantitative estimate of the importance of such effects may be obtained by using some of the results of mulllpleascattering thoory. 13 This appendix. shows the calculations for the angular distribution of outgoing deuterons that result rom pickup by luelastically ocattered protons.

In $\mathrm{Eq}$. (13) of Part I, we have approximated the exact wave function $\left(\psi^{(t)}\right)$ describing the proton-initial-nucleus system by the elastic wave function $\delta^{(+)} \mathrm{CP} \varphi_{i}$. We can write formally

$$
\psi^{(t)}=\Omega^{(+)} \varphi_{i}=\dot{F} \Omega^{(t)} \mathrm{CP} \varphi_{i} \cdot,
$$

where $F$ is an operator that can be thought of as describing the inelastic processes. (In Eq. (13), F = 1.) Following Watson, ${ }^{13} \mathrm{~F}$ can be expressed formally in terms of the two-body $t$ matrices:

$$
\begin{aligned}
& F=1+\frac{1}{a^{(t)}-V_{C P}} \sum_{\alpha=1}^{A} P_{0} t_{\alpha} F_{\alpha} \\
& F_{\alpha}=1+\frac{1}{a^{(t)}-V_{C P}} \sum_{\beta \neq \alpha}^{A} P_{0} t_{\beta} F_{\beta}
\end{aligned}
$$

which gives a perturbation type solution for $F$. Here $P_{0}$ is an operator that picks out only the inelastic part of $t$, i.e., it is 
zero for all elastic. two-body scatterings. To first order in the $t_{\alpha}$, Eq. (1) becomes

$$
\psi^{(t)} \simeq e^{i n \vec{k}_{0} \cdot \vec{r}}+\frac{1}{a^{(t)}-v_{C P}} P_{O} \sum_{\alpha} t_{\alpha} e^{i n \vec{k}_{0} \cdot \vec{r}}
$$

Referring to Eq. (27) of Part II, we may write

$$
M=M_{0}+M_{1},
$$

where $M_{0}$ is Eq. (29) and $M_{1}$ is the first-order inelastic correction term.

$M_{1}=\int d \vec{k} \phi^{*}(k) \Phi\left(\vec{k}+n_{2} \frac{\vec{K}}{2}\right) \int d \vec{r}_{P} e^{i\left(\vec{k}-n_{2} \frac{\vec{K}}{2}\right) \cdot \vec{r}_{P}} \frac{l}{a^{(+)}-v_{C P}} P_{0} \sum_{\alpha} t_{\alpha} e^{i n \vec{k}_{0} \cdot \dot{\vec{r}}_{P}}$

Expanding the energy denominator in a complete set of states designated by $k$, we find

$$
\begin{aligned}
M_{1}=\frac{2 M^{*}}{\hbar} \int d \vec{k} \phi^{*}(k) \Phi\left(\vec{k}+n_{2} \frac{\vec{k}}{2}\right) \int d \vec{r}_{P} e^{i\left(\vec{k}-n_{2} \frac{\vec{k}}{2}\right) \cdot \vec{r}_{P}} \\
\quad \times \int d \vec{k} e^{i \vec{k} \cdot \vec{r}_{P}} \frac{P_{0}}{n^{2} k_{0}^{2}-k^{2}+i \epsilon} \int d \vec{r}_{P}^{\prime} e^{-i \vec{k}^{2} \cdot \vec{r}^{\prime} P} \sum_{\alpha} t_{\alpha} e^{i n \vec{k}_{0} \cdot \vec{r}_{P}} .
\end{aligned}
$$

If we now assume a large homogeneous collection of massive scatterers, the last integral becomes

$$
\begin{gathered}
I=\int d \vec{r}_{\mathrm{P}^{\prime}} e^{-i \vec{k} \cdot \vec{r}_{P^{\prime}}} \sum_{\alpha} t_{\alpha} e^{i n \vec{k}_{0} \cdot \vec{r}_{\mathrm{P}}^{\prime}}=\sum_{\alpha}\left(\vec{k}\left|t_{\alpha}\right| n \vec{k}_{0}\right) \\
\cong \sum_{\alpha} e^{-i\left(\vec{k}-n \vec{k}_{0}\right) \cdot \vec{z}_{\alpha}}\left(\vec{k}|t| n \vec{k}_{0}\right)
\end{gathered}
$$


where the $\alpha$ dependence has been removed from the two-body: scattering amplitude, $\left(\vec{k}|\mathrm{t}| \mathrm{n} \overrightarrow{\mathrm{k}}_{0}\right)$, and $\vec{z}_{\alpha}$ is the coordinate of the ath sccatterer. We next approximate

$$
\sum_{\alpha}=\rho_{0} \int \underset{\mathrm{dz}_{\alpha}}{ }
$$

where $\rho_{0}$ is $A / \frac{4}{3} \pi R_{0}{ }^{3}$. Equation (7) then hesomes

$I=3 A \frac{j_{1}\left(\left|\vec{k}-n \vec{k}_{0}\right| R_{0}\right)}{\left|\vec{k} \cdot n \cdot \vec{k}_{0}\right| R_{0}}\left(\vec{k}|t| n \vec{k}_{0}\right)=f\left(\vec{k}, n \vec{k}_{0}\right)$.

The integral over $k$ may be performed in $\mathrm{Eq} .(6)$, giving a result like Eq. (29) of Part II. Then we have

$M_{1}=\frac{6 M^{*} A}{k^{2}} \int d \vec{k} M_{0}(\vec{k}, \vec{k}) \frac{1}{n^{2}, k_{0}^{2}-k^{2}+i \epsilon} P_{0} f\left(\vec{k}, n \vec{k}_{0}\right)$.

The form of $\mathrm{M}_{9}$ above allows a physical interpretation. The term $P_{O} \mathrm{f}\left(\vec{k}, \mathrm{n} \overrightarrow{\mathrm{k}}_{0}\right)$ describes protons that have scattered into an intermediate state $\vec{\kappa}$. The factor $\left(\vec{k}|\dot{t}| n \vec{k}_{0}\right)$ gives the distribution of these intermediatestate protons, and $\frac{\vec{j}_{1}\left(\left|\vec{k}-n \vec{k}_{0}\right| R_{0}\right)}{\left|\vec{k}-n \vec{k}_{0}\right| R_{0}}$ shows that they set up an interference pattern due to the finfte nuclear size. Finally, $P_{0}$ stipulates that only the inelastic terms of $\left(\vec{k}|t| n \vec{k}_{0}\right)$ are to be used. These particles then propagate in a dispersive medium, described by the energy denominator, and $M_{0}$ gives the amplitude for forming an outgoing deuteron. The integration is carried out over all possible values of $\vec{\kappa} \circ$ The energy denominator can be written 


$$
\frac{1}{n^{2} k_{0}{ }^{2}-k^{2}+i \epsilon}=-1 \pi 8\left(n^{2} k_{0}{ }^{2}-k^{2}\right)+P V\left(\frac{1}{n^{2} k_{0}{ }^{2}-k^{2}}\right) \text {, }
$$

where PV indicates we are to take the principal value of the integral. The delta function may be directly integrated to yield

$$
-\frac{6 \mathrm{M}^{*} \mathrm{~A}}{\hbar^{2}} \frac{i \pi}{2} n \mathrm{k}_{0} \int d \Omega \quad \mathrm{M}_{0}(\overrightarrow{\mathrm{k}}, \vec{k}) \mathrm{P}_{0} f\left(\vec{k}, \mathrm{n} \overrightarrow{\mathrm{k}}_{0}\right)
$$

where the integral is over all angles of $\vec{k}$ and $|\vec{k}|=\left|\mathrm{n} \overrightarrow{\mathrm{k}}_{0}\right|$. In the usual treatment of the principal-value integral, 39 the approximation is made that the particle travels a long distance before the next scattering, or before the particle is observed. In these treatments, however, the angular integration is performed before the principal-value integral is calculated, so that an integral such as

$$
\int_{0}^{\infty} \frac{F(k) e^{i k r} d k}{k_{0}^{2}-k^{2}} \simeq F\left(k_{0}\right) \int_{0}^{\infty} \frac{e^{i k r}}{k_{0}^{2}-k^{2}} d k
$$

is considered. For large $r$, or $\mathrm{kr}>>1$, the main contribution to this integral comes at $k=k_{0}$, and the principal-value integral gives the same result as the delta-function integral. However, since we wish to perform the angular integration later, we must evaluate an integral of the form

$$
\int_{0}^{\infty} \frac{F(k) e^{i k r \cos \theta}}{k_{0}^{2}-k^{2}} d k
$$


The region where $F(k)$ cannot be taken out of the integral is $\cos \theta \leqslant \frac{1}{\mathrm{kr}}$, and in the limit of very large $r$, this region becomes vanishingly small.

If $\int_{0}^{\infty} \frac{F(k)}{k_{0}^{2}-k^{2}} d k$ is finite, then this region does not contribute to Ey. (13) abovc, and $F(k)$ may he taken outside the integral. Since this turns out to be the case for our integrals, we can write

$M_{1}=-\frac{6 \pi i M^{*} A n k_{0}}{\hbar^{2}} \int d s M_{0}(\vec{K}, \vec{k}) P_{0} f\left(\vec{k}, n \vec{k}_{0}\right)$

in the limit of large distances between the scattering and pickup. If $\alpha$ is the angle between $\vec{k}_{0}$ and $\vec{K}$, and $\theta$, the angle between $\vec{k}_{0}$ and $\vec{k}$, then the cosine of the angle between $\vec{k}$ and $\vec{k}$ 1s $(\cos \theta \cos \alpha-\sin \theta \sin \alpha \cos \varnothing)$, where $\theta$ and $\varnothing$ are angles in the integration over $d s$. Since we have assumed a Gaussian form for $M_{0}$, the integration of Eq. (14) is of the form

$$
\partial=\int_{0}^{2 \pi} \int_{0}^{\pi} \sin \theta d \theta d \phi e^{a(\cos \theta \cos \alpha \cdot \sin \theta \sin \alpha \cos \varnothing)} g(\cos \theta) .
$$

For many analytic forms of $g(\cos \theta)$, this integral can be solved in terms of Bessel functions. 40

The results of calculations are shown in Fig. 1, where it is assumed that there is no interference between the elastic and inelastic terms, $M_{0}$ and $M_{1}$. 
APPENDIX II. JUSTIFICATION FOR EQUATTON (14), PART II

In Part II (see Eqs. (13), (14), and (15)) a solution was obtained for the coherent outgoing deuteron wave function, $\delta_{\mathrm{S}}^{(-)}{ }_{\mathrm{CN}} \varphi_{f}$, by assuming that the deuteron size is much smaller than the nuclear radius, or

$$
\bar{r} / R_{0} \ll I .
$$

Here $\vec{r}$ is one-half the average neutron-proton separation in the deuteron, and is charscterized by a length $1 / 2 \alpha$ where $\alpha^{2}=2 m \mathrm{~B} / \mathrm{h}^{2}$. The nuclear radius $R_{0}$ may be expressed as $R_{0}=r_{0} A^{l / 3}$, where $r_{0}=1.5 \times 10^{-13} \mathrm{~cm}$. Then the condition stated in (1) may be written

$$
\text { A } \gg 3 .
$$

One might believe, then, that the calculations of the pickup cross sections with the formulae of Parts II and III are valid for all but light nuclei. However, the approximation of Eq. (14), Part II, introduces two more points that require further investigation.

(a) Although $V_{C N D}(\vec{R})$ was substituted for $V_{C N}\left(\vec{r}_{N V}\right)$ in Eq. (13), the solution of this equation, now a function of $\vec{R}$, is later integrated over the $\vec{r}_{N}$ coordinate rother than $\vec{R}$ 。 The coordinates $\vec{R}$ and $\vec{r}_{N}$ seem to be used interchangeably in the evaluation of the matrix element. Some of the confusion that may exist can be cleared up by enumerating the approximations: (1) the potential $V_{\text {CN }}\left(\vec{r}_{N N}\right)$ determines the behavior of the outgoing deuteron, and exists inside the region $r_{N}<R_{0}$; (ii) it is expedient, however, to assume that the region $r_{N}<R_{O}$ can be approximated by the region. $R<R_{0}$ in order to solve for $\delta_{6}^{(\infty)} \mathrm{CN} \varphi_{f}$ in Eq. (13); (iii) this wave function is finally integrated in Eq. (26) 
over the region $r_{N}<R_{O}$, just as if the approximation in (ii) above had never been made. To justify this last step, it is necessary to show that the integration over $\vec{r}_{\mathrm{N}}$ does not depend critically on small changes (on the order of $\vec{r}$ ) in the location on the nuclear surface.

(b) We have neglected contributions from the region $r_{N}>R_{0}$ (or $R:>R_{U}$ ) in our matrix elemenls. To justify this, we may rxliull the argument of the paragraph above: if the result of the integration over $\vec{r}_{N}$ does not critically depend on the location of the nuclear surface in the region $\infty>r_{N}>R_{0}$, then there is little contribution to the integral from this region. .

We may take into account both points (a) and (b) by showing that the $\vec{r}_{N}$ integration for $r_{N}<R_{0}=\bar{r}$ obtains the same results as the Fourier transform. Let

$$
G(\vec{k})=\int_{r_{N}<R_{1}} e^{i \vec{k} \cdot \vec{r}_{N}} e^{-r^{2} N / 4 a} d \vec{r}_{N},
$$

where $x_{i}\left(\vec{r}_{N}\right)$ has the Gaussian form introduced earlier, and $R_{1}=R_{0}-\bar{r}$. Performing the integration, we find

$$
\begin{aligned}
G(\vec{k})= & 8(a)^{3 / 2} \pi e^{-k^{2} a}\left[\operatorname{Erf}\left(\frac{R_{1}}{2 \sqrt{a}}+i k \sqrt{a}\right)+\operatorname{Erf}\left(\frac{R_{1}}{2 \sqrt{a}}-i k \sqrt{a}\right)\right] \\
& +8 \pi a e^{-R_{1}^{2} / 4 a} \frac{\sin \left(k R_{1}\right)}{k}
\end{aligned}
$$

For

$$
R_{1}^{2} \subsetneq 4 a
$$

the second term of Eq. (14) can be neglected, and the error functions 
In the first term combine to give. $\sqrt{\pi} ; \mathrm{G}(\vec{k})$ then becomes

$$
G(\vec{k})=8(\pi \varepsilon)^{3 / 2} e^{-k^{2} a},
$$

which is just the Fourier transform of the neutron wave function $\left(R_{1}=\infty\right)$. For the case of a $\mathrm{C}^{12}$ nucleus with a nuclearmomentum distribution of $\exp (-\mathrm{E} / 20), 4 \mathrm{a}=2.6 \times 10^{-26} \mathrm{~cm}^{2}$ and $\mathrm{R}_{1}^{2} \simeq 2.0 \times 10^{-26} \mathrm{~cm}^{2}$. The apparent difficulty hereasi.e, that light nuclel such as $c^{12}$ do not satisfy Conditions (2) and (5)-ois compensated in part by the facts that (a) the finalostate deuteron scattering is most important for its absorption effects, and contributes little structure to the angular distribution, and (b) opticalmodel corrections in general become less important for light nuclei. 


\section{REFERENCES}

1. R。 Serber, Phys. Rev。 72, 1114 (1947)。

2. B. Lippmann and Jo Schwinger, Phys。Rev。 79, 469 (1950)。

3. A. C. Helmholz, E.M. MoMillan, and D.C. Sewell, Phys.Rev。 72, $1003(1947)$.

4. R. Serber, Phys. Kev。 tę 1000 (194\%):

5. H.B. Burrows, W. M. Gibson, and J。 Rotblat, Phys.Rev. 80, 1095 (1950)。

6. S. T. Butler, Proc。Roy。Soc。(Iondon) 208, 559. (195I) \& A. B. Bhatia, K. Huang, R. Huby, and H.C.Newns, Phil。Mag. 43, 485 (1952)。

7. E. Gerjouy, Phys. Rev. 91, 645 (19.53)。

8. N. C. Francis and K. M. Watson, Phys。Rev. 93, 313 (1954).

9. M. Gell-Mann and M. L。 Coldberger, Phys.Rev。 9l, 398 (1953)。

10. W. Tobocman and M。H. Kalos, Phys. Rev。 97,132 (1955)。

11. R。 Huby, Prog。 in Nuclear Phys。 3, 177 (1953)。

12. S. T. Butler and O. H. Hittmair, Nuclear Strlpulus Reactions (Wilcy, New York, 1957).

13. K。 M.Watson, Phys。Rev。 205, 1388 (1957).

14. S. Fernbach, R。 Serber, and T。 B. Taylor, Phys. Rev。 75, 1352 (1949).

15. W. B. Cheston, Phys. Rev. 96, 1590 (1954); J。 Sawicki, Phys 。 Rev. 106, $172(1957)$.

16. G. F。 Chew and M. L。 Goldberger, Fhys. Rev。 77, 470 (1950).

17. W. Selove, Phys. Rev. 101, 231 (1956).

18. P.F. Cooper (thesis), Haxvard University (1958) (to be published).

19. R. H. Dalitz, Proc. Phys. Soc. (London) A66, 28 (1953).

20. A.C. Juveland and W. Jeatschke, Phys. Rev. 110, 456 (1958).

21. H. C. Newns, Proc. Phys.Soc。(London) A66, 477 (1953)。 
22. J. Horowitz and A. M. L. Messiah, J。Phys。Rad。 14, 695 (1953).

23. 0. Hittmair, Z. Physik 144, 449 (1956).

24. B. J. Malenka, Phys。Rev。98, 843 (1955)。

25. L. Wolfenstein and J.Ashkin, Phys. Rev. 85, 947 (1952); L. Wolfenstein, Annual Review of Nuclear Science 6, 43 (1956).

26. The author would like to express his appreciation to Professor Gooffrey Chew far hringing up this point, and to Dr. Leonard Rodberg for his help and interest in the derivation of the formalism.

27. B. Lippmann, Phys. Rev. 102, 254 (1956)。

28. I. I. Schiff, Quantum Mechanics (McGraw $-H i 11$, New York, 1949), p. 184.

29. R. Hart and E.Montroll, J.Appl. Phys。22, 376 (1951); E. Montroll and J.M. Greenberg, Phys. Rev。 86, 889 (1952).

30. A. Glassiold, Revi. Modcrn Phys. (in press).

31. J.M. Wilcox ana B. J. Moyer, Phys. Rev. 99, 875 (1955).

32. J. B. Cladis, W.N.Hess, and B. J. Moyer, Phys.Rev.87, 425 (1952).

33. S. Fernbach, W. Heckrotte, and J.V. Lepore, Phys.Rev. 27, 1059 (1955)。

34. E. Fermi, Nuovo cimento 11, 407 (1954)。

35. E. Fermi, Nuovo cimento Supp. 10, 17 (1955).

36. W. Lakin, Phys。Rev. 98, 139 (1955).

37. H. P. Stapp, Phys. Rev。 107, 607 (1957)。

38. J. Blatt and V. Weisskopf, Theoretical Nuclear Physics (Wiley, New York, 1952)。

39. P. A. M. Dirac, The Principles of Quantum Mechanics (Clarendon, Oxford, 1947), p. 193.

40. G. N. Watson, Bessel Functions (Mecmillan, New York, 1948). 